\title{
The truncated EM method for stochastic differential equations with Poisson jumps
}

\author{
Shounian Denga, ${ }^{\mathrm{a} b}$, Weiyin Fei ${ }^{\mathrm{b}, *}$, Wei Liu ${ }^{\mathrm{c}}$, Xuerong Mao ${ }^{\mathrm{d}}$ \\ ${ }^{a}$ School of Science, Nanjing University of Science and Technology, Nanjing, Jiangsu 210094, China \\ ${ }^{b}$ School of Mathematics and Physics, Anhui Polytechnic University, Wuhu, Anhui 24100, China \\ ${ }^{c}$ Department of Mathematics, Shanghai Normal University, Shanghai 200234, China \\ ${ }^{d}$ Department of Mathematics and Statistics, University of Strathclyde, Glasgow G1 1XH, U.K.
}

\begin{abstract}
In this paper, we use the truncated Euler-Maruyama (EM) method to study the finite time strong convergence for SDEs with Poisson jumps under the Khasminskii-type condition. We establish the finite time $\mathcal{L}^{r}(r \geq 2)$-convergence order when the drift and diffusion coefficients satisfy the super-linear growth condition and the jump coefficient satisfies the linear growth condition. The result shows that the optimal $\mathcal{L}^{r}$-convergence order is close to 1 . This is significantly different from the result on SDEs without jumps. When all the three coefficients of SDEs are allowing to grow super-linearly, the $\mathcal{L}^{r}(0<r<2)$-convergence results are also investigated and the optimal $\mathcal{L}^{r}$-convergence order is shown to be not greater than $1 / 4$. Moreover, we prove that the truncated EM method preserves nicely the mean square exponential stability and asymptotic boundedness of the underlying SDEs with Piosson jumps. Several examples are given to illustrate our results.
\end{abstract}

Keywords: Stochastic differential equations, local Lipschitz condition, Khasminskii-type condition, truncated EM method, Piosson jumps.

\section{Introduction}

Due to the broad applications in modeling uncertain phenomenon, stochastic differential equations (SDEs) driven by Brownian motions have been attracting lots of attentions [1, 2, 3]. When some unexpected events happen, some jumps may be needed to model the effects of those 5 events. For example, a breaking news after the close of the stock market may lead to a huge difference between today's closing price and tomorrow's opening price. To take both the continuous and discontinuous random effects into consideration, SDEs driven by both Brownnian motions and Poisson jumps are often employed as a generalisation of the SDEs only driven by Brownian motions.

Despite the wide applications, the explicit solutions to SDEs are hardly found. Therefore, to construct some efficient numerical methods is of extremely important. The series works of Higham and Kloeden [4, 5, 6] studied some implicit methods for SDEs with Poisson jumps. In their papers, the strong convergence, the convergence rates and stability of different implicit

\footnotetext{
${ }^{*}$ Corresponding author

Email address: wyf ei@ahpu.edu.cn (Weiyin Fei )
} 
methods were proposed and investigated for some SDEs, whose drift coefficient satisfies non-

15 global Lipschitz condition, and both the diffusion coefficient and the coefficient for the Poisson jumps are global Lipschitzian. When the global Lipschitz condition on the diffusion coefficient is disturbed, the tamed EM and the tamed Milstein methods were proposed for SDEs driven by the more generalised process, Lévy process [7, 8]. The taming techniques were original proposed in [9] for the construction of explicit methods for SDEs with non-globally Lipschitz

20 continuous coefficients. As indicated in [10], explicit methods have their own advantages on the relatively simple structure and the avoidance of solving some nonlinear systems in each iteration. Therefore, the studies on explicit methods for SDEs with non-globally Lipschitz coefficients have been blooming in recent years. Sine and cosine functions were employed in [11] to construct some explicit methods for SDEs with both the drift and diffusion coefficients growing super-

25 linearly. The taming techniques were modified and generalised in [12] and [13]. The truncated EM method was proposed in [14, 15]. The partially truncated EM scheme can be found in [16] and [17].

In this paper, we borrow the truncating idea to propose the truncated EM method for SDEs with Poisson jumps. The main contributions of this work are twofold. Firstly, all the drift 30 coefficient, the diffusion coefficient and the coefficient for Poisson jumps are allowed to grow super-linearly. To our best knowledge, this is the first work to study an explicit numerical method for SDEs with all the three coefficients that can grow super-linearly. Secondly, both the finite time convergence and asymptotic behaviours of the method are investigated.

It should be noted that the truncated EM scheme for SDEs with the global Lipschitzian pure

35 jumps was studied in [18]. Other numerical methods for SDEs with Poisson jumps or Lévy process were also proposed and investigated in [19, 20, 21, 22, 23], we just mention some of them here and refer the readers to the references therein. For the detailed and systemic introductions to numerical methods for SDEs and SDEs with jump, we refer the readers to the monographs [24] and [25].

40 This paper is constructed as follows. In Section 2, we introduce some necessary mathematical preliminaries. Section 3 contains the main results on the finite time convergence. The asymptotic behaviours, stability and boundedness, of the numerical solutions are presented in Section 4. Several examples are given in the Section 5. Section 6 concludes the paper and points out some future research.

\section{2. Mathematical Preliminaries}

Throughout this paper, unless otherwise specified, let $(\Omega, \mathcal{F}, \mathbb{P})$ be a complete probability space with a filtration $\left\{\mathcal{F}_{t}\right\}_{t \geq 0}$ satisfying the usual conditions (i.e., it is increasing and right continuous while $\mathcal{F}_{0}$ contains all $\mathbb{P}$-null sets). Let $\mathbb{E}$ denote the probability expectation with respect to $\mathbb{P}$. Let $B(t)$ be an $m$-dimensional Brownian motion defined on the probability space and is

$50 \mathcal{F}_{\mathrm{t}}$-adapted. $N(t)$ is a scalar Poisson process independent of $B(t)$ with the compensated Poisson precess $\widetilde{N}(t)=N(t)-\lambda t$, where the parameter $\lambda$ is the jump intensity. If $A$ is a vector or matrix, its transpose is denoted by $A^{T}$. If $x \in \mathbb{R}^{d}$, then $|x|$ is the Euclidean norm. If $A$ is a matrix, its trace norm is denoted by $|A|=\sqrt{\left(A^{T} A\right)}$. For two real numbers $a$ and $b$, we use $a \vee b=\max (a, b)$ and $a \wedge b=\min (a, b)$. For a set $G$, its indicator function is denoted by $\mathbb{I}_{G}$. Moreover, $\mathcal{L}^{r}=\mathcal{L}^{r}(\Omega, \mathcal{F}, \mathbb{P})$

55 denotes the space of random variables $X$ with a norm $|x|_{r}:=\left(\mathbb{E}|X|^{r}\right)^{1 / r}<\infty$, for $r>0$. In what follows, for notational simplicity, we use the convention that $C$ represents a generic positive constant, the value of which may be different for different appearances. 
Consider a $d$-dimensional SDE with Piosson jumps:

$$
d x(t)=f(x(t)) d t+g(x(t)) d B(t)+h\left(x\left(t^{-}\right)\right) d N(t), \quad t \geq 0 .
$$

with the initial value $x(0)=x_{0} \in \mathbb{R}^{d}$, where $x\left(t^{-}\right)$denotes $\lim _{s \rightarrow t^{-}} x(s)$. Here, $f: \mathbb{R}^{d} \rightarrow \mathbb{R}^{d}$ is the drift coefficient, $g: \mathbb{R}^{d} \rightarrow \mathbb{R}^{d \times m}$ is the diffusion coefficient, $h: \mathbb{R}^{d} \rightarrow \mathbb{R}^{d}$ is the jump coefficient.

\section{3. Finite time convergence}

\subsection{Convergence rate of the partially truncated EM method in $\mathcal{L}^{r}(r \geq 2)$}

In order to discuss the convergence order of the truncated EM method in $\mathcal{L}^{r}$ for $r \geq 2$. We assume that $f$ and $g$ can be decomposed as $f(x)=F_{1}(x)+F(x)$ and $g(x)=G_{1}(x)+G(x)$, where $F_{1}, F: \mathbb{R}^{d} \rightarrow \mathbb{R}^{d}$, and $G_{1}, G: \mathbb{R}^{d} \rightarrow \mathbb{R}^{d \times m}$. Moreover, the coefficients $F, G, F_{1}, G_{1}$ and $h$ satisfy 65 the following conditions.

Assumption 3.1. There exist constants $L_{1}>0$ and $\gamma \geq 0$ such that

$$
\begin{array}{r}
\left|F_{1}(x)-F_{1}(y)\right| \vee\left|G_{1}(x)-G_{1}(y)\right| \vee|h(x)-h(y)| \leq L_{1}|x-y|, \quad \forall x, y \in \mathbb{R}^{d}, \\
|F(x)-F(y)| \vee|G(x)-G(y)| \leq L_{1}\left(1+|x|^{\gamma}+|y|^{\gamma}\right)|x-y|, \quad \forall x, y \in \mathbb{R}^{d},
\end{array}
$$

where the parameter $\gamma$ is called the super-linear growth constant. By Assumption 3.1. we can derive that there exists a positive constant $K_{1}$ such that

$$
\left|F_{1}(x)\right| \vee\left|G_{1}(x)\right| \vee|h(x)| \leq K_{1}(1+|x|), \quad \forall x \in \mathbb{R}^{d},
$$

which implies that $F_{1}, G_{1}$ and $h$ satisfy the linear growth condition. Similarly, we have

$$
|F(x)| \vee|G(x)| \leq\left(2 L_{1}+|F(0)|+|G(0)|\right)|x|^{1+\gamma}, \quad \forall|x| \geq 1 .
$$

Assumption 3.2. There exists a pair of constants $\bar{r}>2$ and $L_{2}>0$ such that

$$
(x-y)^{T}(F(x)-F(y))+\frac{\bar{r}-1}{2}|G(x)-G(y)|^{2} \leq L_{2}|x-y|^{2}, \quad \forall x, y \in \mathbb{R}^{d} .
$$

By Assumption 3.2 we can derive that for any $r \in(2, \bar{r})$

$$
(x-y)^{T}(f(x)-f(y))+\frac{r-1}{2}|g(x)-g(y)|^{2} \leq L_{3}|x-y|^{2} .
$$

where $L_{3}=2 L_{1}+L_{2}+\frac{L_{1}^{2}+(r-1)(\bar{r}-1)}{\bar{r}-r}$ (see [16]).

Assumption 3.3. (Khasminskii-type condition) There exist constants $\bar{p}>\bar{r}$ and $K_{2}>0$ such that

$$
x^{T} F(x)+\frac{\bar{p}-1}{2}|G(x)|^{2} \leq K_{2}\left(1+|x|^{2}\right), \quad \forall x \in \mathbb{R}^{d} .
$$


By Assumption 3.3 we also have that for any $p \in(2, \bar{p})$

$$
x^{T} f(x)+\frac{p-1}{2}|g(x)|^{2} \leq K_{3}\left(1+|x|^{2}\right)
$$

where $K_{3}=2 K_{1}+K_{2}+\frac{K_{1}^{2}+(p-1)(\bar{p}-1)}{\bar{p}-p}$ (see [16]).

The truncated idea is to deal with super-linearly growing coefficients. In the viewpoint of the finite time convergence, the linearly growing coefficient does not cause any problem to the EM scheme and hence there is no need to truncate it [16]. In our truncated EM method, we only truncate the super-linearly growing terms, that is $F$ and $G$. To define the truncated EM scheme, we first choose a strictly increasing function $\mu: \mathbb{R}^{+} \rightarrow \mathbb{R}^{+}$such that $\mu(n) \rightarrow \infty$, as $n \rightarrow \infty$ and

$$
\sup _{|x| \leq n}|F(x)| \vee|G(x)| \leq \mu(n), \quad \forall n \geq 1 .
$$

The inverse function of $\mu$ is denoted by $\mu^{-1}$. For some $\varepsilon \in(0,1 / 4]$, we define a strictly decreasing function $\varphi:(0,1] \rightarrow(0, \infty)$

$$
\varphi(\Delta)=\hat{h} \Delta^{-\varepsilon}, \quad \forall \Delta \in(0,1]
$$

where $\hat{h} \geq 1$ is a constant. Hence, we get

$$
\lim _{\Delta \rightarrow 0} \varphi(\Delta)=\infty \quad \text { and } \quad \Delta^{1 / 4} \varphi(\Delta) \leq \hat{h}, \quad \forall \Delta \in(0,1] .
$$

For a given step size $\Delta \in(0,1]$, let us define a mapping $\pi_{\Delta}$ from $\mathbb{R}^{d}$ to the closed ball $\left\{x \in \mathbb{R}^{d}\right.$ : $\left.|x| \leq \mu^{-1}(\varphi(\Delta))\right\}$ by

$$
\pi_{\Delta}=\left(|x| \wedge \mu^{-1}(\varphi(\Delta))\right) \frac{x}{|x|} .
$$

We set $x /|x|=0$ when $x=0$. We then define the partially truncated functions:

$$
\begin{array}{r}
F_{\Delta}(x)=F\left(\pi_{\Delta}(x)\right), \quad G_{\Delta}(x)=G\left(\pi_{\Delta}(x)\right), \quad \forall x \in \mathbb{R}^{d} \\
f_{\Delta}(x)=F_{1}(x)+F_{\Delta}(x) \text { and } g_{\Delta}(x)=G_{1}(x)+F_{\Delta}(x), \quad \forall x \in \mathbb{R}^{d} .
\end{array}
$$

It is useful to see that

$$
\left|F_{\Delta}(x)\right| \vee\left|G_{\Delta}(x)\right| \leq \varphi(\Delta), \quad \forall x \in \mathbb{R}^{d},
$$

which means that $F_{\Delta}$ and $G_{\Delta}$ are bounded while $F$ and $G$ may not. The following lemma shows that the truncated functions maintain the Khaminskii-type condition nicely (see Lemma 2.5, [26]).

Lemma 3.4. Let Assumption 3.3 hold. Then, for all $\Delta \in(0,1]$,

$$
x^{T} F_{\Delta}(x)+\frac{\bar{p}-1}{2}\left|G_{\Delta}(x)\right|^{2} \leq 2 K_{2}\left[1 \wedge 1 / \mu^{-1}(\varphi(1))\right]\left(1+|x|^{2}\right), \quad \forall x \in \mathbb{R}^{d} .
$$

For any $p \in(2, \bar{p})$, we also have

$$
x^{T} f_{\Delta}(x)+\frac{p-1}{2}\left|g_{\Delta}(x)\right|^{2} \leq K_{4}\left(1+|x|^{2}\right), \quad \forall x \in \mathbb{R}^{d},
$$


where $K_{4}=2 K_{1}+2 K_{2}\left[1 \wedge 1 / \mu^{-1}(\varphi(1))\right]+\frac{K_{1}^{2}+(p-1)(\bar{p}-1)}{\bar{p}-p}$ (see [16]). From now on, we will fix $T>0$ arbitrarily. Let $M$ be a positive integer. We take step size $\Delta=T / M \in(0,1]$. For any $0 \leq t \leq T$, we define

$$
\kappa(t)=\lfloor t / \Delta\rfloor \Delta
$$

where $\lfloor t / \Delta\rfloor$ denotes the integer part of $t / \Delta$. Then we form the discrete-time truncated EM solutions $X_{\Delta}\left(t_{k}\right) \approx x\left(t_{k}\right)$, for $t_{k}=k \Delta$ by setting $X_{\Delta}(0)=x_{0}$ and computing

$$
X_{\Delta}\left(t_{k+1}\right)=X_{\Delta}\left(t_{k}\right)+f_{\Delta}\left(X_{\Delta}\left(t_{k}\right)\right) \Delta+g_{\Delta}\left(X_{\Delta}\left(t_{k}\right)\right) \Delta B_{k}+h\left(X_{\Delta}\left(t_{k}^{-}\right)\right) \Delta N_{k}, \quad 0 \leq k \leq M-1,
$$

where $\Delta B_{k}=B\left(t_{k+1}\right)-B\left(t_{k}\right), \Delta N_{k}=N\left(t_{k+1}\right)-N\left(t_{k}\right)$. For $0 \leq t \leq T$, it is consentient to use the continuous-time step process $\bar{x}_{\Delta}(t)$ which is defined by

$$
\bar{x}_{\Delta}(t)=\sum_{k=0}^{M-1} X_{\Delta}\left(t_{k}\right) \mathbb{I}_{\left[t_{k}, t_{k+1}\right)}(t)
$$

where $\mathbb{I}$ is an indicator function. The other continuous-time process is defined by

$$
x_{\Delta}(t)=x_{0}+\int_{0}^{t} f_{\Delta}\left(\bar{x}_{\Delta}(s)\right) d s+\int_{0}^{t} g_{\Delta}\left(\bar{x}_{\Delta}(s)\right) d B(s)+\int_{0}^{t} h\left(\bar{x}_{\Delta}\left(t^{-}\right)\right) d N(s) .
$$

It is easy to see that $x_{\Delta}\left(t_{k}\right)=\bar{x}_{\Delta}\left(t_{k}\right)=X_{\Delta}\left(t_{k}\right)$. Moreover, $x_{\Delta}(t)$ is an Itô process satisfying Itô differential

$$
d x_{\Delta}(t)=f_{\Delta}\left(\bar{x}_{\Delta}(t)\right) d t+g_{\Delta}\left(\bar{x}_{\Delta}(t)\right) d B(t)+h\left(\bar{x}_{\Delta}\left(t^{-}\right)\right) d N(t) .
$$

We first state a known result (see [7]) as a lemma.

Lemma 3.5. Let Assumption 3.1 and 3.3 hold. Then the SDE 2.1) has a unique global solution $x(t)$. Moreover, for any $p \in(2, \bar{p})$,

$$
\sup _{0 \leq t \leq T} \mathbb{E}|x(t)|^{p}<\infty, \quad \forall T>0 .
$$

In order to bound the $p$-th moment of the truncated EM solution, we need the following lemma.

Lemma 3.6. For any $\Delta \in(0,1]$ and $0 \leq t \leq T$,

$$
\begin{aligned}
& \mathbb{E}\left(\left|x_{\Delta}(t)-\bar{x}_{\Delta}(t)\right|^{\hat{p}} \mid \mathcal{F}_{\kappa(t)}\right) \leq C\left((\varphi(\Delta))^{\hat{p}} \Delta^{\hat{p} / 2}+\Delta\right)\left(1+\left|\bar{x}_{\Delta}(t)\right|^{\hat{p}}\right), \quad \hat{p} \geq 2, \\
& \mathbb{E}\left(\left|x_{\Delta}(t)-\bar{x}_{\Delta}(t)\right|^{\hat{p}} \mid \mathcal{F}_{\kappa(t)}\right) \leq C(\varphi(\Delta))^{\hat{p}} \Delta^{\hat{p} / 2}\left(1+\left|\bar{x}_{\Delta}(t)\right|^{\hat{p}}\right), \quad 0<\hat{p}<2 .
\end{aligned}
$$

Proof. Fix any $\hat{p} \geq 2$. By Assumption 3.1 and 3.12, we have

$$
\begin{aligned}
& \mathbb{E}\left(\left|x_{\Delta}(t)-\bar{x}_{\Delta}(t)\right|^{\hat{p}} \mid \mathcal{F}_{\kappa(t)}\right) \\
& =\mathbb{E}\left(\left|\int_{\kappa(t)}^{t} f_{\Delta}\left(\bar{x}_{\Delta}(s)\right) d s+\int_{\kappa(t)}^{t} g_{\Delta}\left(\bar{x}_{\Delta}(s)\right) d B(s)+\int_{\kappa(t)}^{t} h\left(\bar{x}_{\Delta}(s)\right) d N(s)\right|^{\hat{p}} \mid \mathcal{F}_{\kappa(t)}\right) \\
& \leq C\left(\mathbb{E}\left(\left|\int_{\kappa(t)}^{t} f_{\Delta}\left(\bar{x}_{\Delta}(s)\right) d s\right|^{\hat{p}} \mid \mathcal{F}_{\kappa(t)}\right)+\mathbb{E}\left(\left|\int_{\kappa(t)}^{t} g_{\Delta}\left(\bar{x}_{\Delta}(s)\right) d B(s)\right|^{\hat{p}} \mid \mathcal{F}_{\kappa(t)}\right)+\mathbb{E}\left(\left|\int_{\kappa(t)}^{t} h\left(\bar{x}_{\Delta}(s)\right) d N(s)\right|^{\hat{p}} \mid \mathcal{F}_{\kappa(t)}\right)\right) \\
& \left.\leq C\left((\varphi(\Delta))^{\hat{p}}\right) \Delta^{\hat{p} / 2}+\Delta^{p / 2}\left(1+\left|\bar{x}_{\Delta}(t)\right|^{\hat{p}}\right)+\mathbb{E}\left(\left|\int_{\kappa(t)}^{t} h\left(\bar{x}_{\Delta}(s)\right) d N(s)\right|^{\hat{p}} \mid \mathcal{F}_{\kappa(t)}\right)\right)
\end{aligned}
$$


where $C$ is a generic constant, the value of which may change between occurrences. By the characteristic function's argument [27], for $\Delta \in(0,1]$, we have

$$
\mathbb{E}\left|\Delta N_{k}\right|^{\hat{p}} \leq c_{0} \Delta,
$$

where $c_{0}$ is a positive constant which is independent of $\Delta$. Therefore,

$$
\begin{aligned}
& \mathbb{E}\left(\left|\int_{\kappa(t)}^{t} h\left(\bar{x}_{\Delta}(s)\right) d N(s)\right|^{\hat{p}} \mid \mathcal{F}_{\kappa(t)}\right)=\mathbb{E}\left(\left.\left|h\left(x_{\Delta}(\kappa(t))\right) \Delta N_{k}\right|\right|^{\hat{p}} \mid \mathcal{F}_{\kappa(t)}\right) \\
& =\left|h\left(x_{\Delta}(\kappa(t))\right)\right|^{\hat{p}} \mathbb{E}\left|\Delta N_{k}\right|^{\hat{p}} \leq C\left(1+\left|\bar{x}_{\Delta}(t)\right|^{\hat{p}}\right) \Delta .
\end{aligned}
$$

Inserting this into 3.19p and combing with $\Delta^{\hat{p} / 2} \leq \Delta$ gives

$$
\begin{aligned}
\mathbb{E}\left(\left|x_{\Delta}(t)-\bar{x}_{\Delta}(t)\right|^{\hat{p}} \mid \mathcal{F}_{\kappa(t)}\right) & \leq C(\varphi(\Delta))^{\hat{p}} \Delta^{\hat{p} / 2}+C \Delta\left(1+\left|\bar{x}_{\Delta}(t)\right|^{\hat{p}}\right) \\
& \leq C\left((\varphi(\Delta))^{\hat{p}} \Delta^{\hat{p} / 2}+\Delta\right)\left(1+\left|\bar{x}_{\Delta}(t)\right|^{\hat{p}}\right) .
\end{aligned}
$$

When $0<\hat{p}<2$, the Jensen inequality gives

$$
\begin{aligned}
\mathbb{E}\left(\left|x_{\Delta}(t)-\bar{x}_{\Delta}(t)\right|^{\hat{p}} \mid \mathcal{F}_{\kappa(t)}\right) & \leq\left[\mathbb{E}\left(\left|x_{\Delta}(t)-\bar{x}_{\Delta}(t)\right|^{2} \mid \mathcal{F}_{\kappa(t)}\right)\right]^{\hat{p} / 2} \\
& \leq C\left((\varphi(\Delta))^{2} \Delta+\Delta\right)^{\hat{p} / 2}\left(1+\left|\bar{x}_{\Delta}(t)\right|^{\hat{p}}\right) \\
& \leq C\left((\varphi(\Delta))^{\hat{p}} \Delta^{\hat{p} / 2}+\Delta^{\hat{p} / 2}\right)\left(1+\left|\bar{x}_{\Delta}(t)\right|^{\hat{p}}\right) \\
& \leq C(\varphi(\Delta))^{\hat{p}} \Delta^{\hat{p} / 2}\left(1+\left|\bar{x}_{\Delta}(t)\right|^{\hat{p}}\right) .
\end{aligned}
$$

Thus, we complete the proof.

Lemma 3.7. Let Assumption 3.1 and 3.3 hold and let $p \in(2, \bar{p})$ be arbitrary. Then

$$
\sup _{0 \leq \Delta \leq 1} \sup _{0 \leq t \leq T} \mathbb{E}\left|x_{\Delta}(t)\right|^{p} \leq C, \quad \forall T>0,
$$

Proof. Fix any $\Delta \in(0,1]$ and $T>0$. By the Itô formula and (3.13), we have

$$
\begin{aligned}
\mathbb{E}\left|x_{\Delta}(t)\right|^{p}-\left|x_{0}\right|^{p} \leq & \mathbb{E} \int_{0}^{t} p\left|x_{\Delta}(t)\right|^{p-2}\left(x_{\Delta}^{T}(s) f_{\Delta}\left(\bar{x}_{\Delta}(s)\right)+\frac{p-1}{2}\left|g_{\Delta}\left(\bar{x}_{\Delta}(s)\right)\right|^{2}\right) d s \\
& +\lambda \mathbb{E}\left(\int_{0}^{t}\left|x_{\Delta}\left(s^{-}\right)+h\left(\bar{x}_{\Delta}\left(s^{-}\right)\right)\right|^{p}-\left|x_{\Delta}\left(s^{-}\right)\right|^{p}\right) d s \\
\leq & \mathbb{E} \int_{0}^{t} p\left|x_{\Delta}(t)\right|^{p-2}\left(\bar{x}_{\Delta}^{T}(s) f_{\Delta}\left(\bar{x}_{\Delta}(s)\right)+\frac{p-1}{2}\left|g_{\Delta}\left(\bar{x}_{\Delta}(s)\right)\right|^{2}\right) d s \\
& +\mathbb{E} \int_{0}^{t} p\left|x_{\Delta}(s)\right|^{p-2}\left(x_{\Delta}(s)-\bar{x}_{\Delta}(s)\right)^{T} f_{\Delta}\left(\bar{x}_{\Delta}(s)\right) d s \\
& +\lambda \mathbb{E}\left(\int_{0}^{t}\left|x_{\Delta}\left(s^{-}\right)+h\left(\bar{x}_{\Delta}\left(s^{-}\right)\right)\right|^{p}-\left|x_{\Delta}\left(s^{-}\right)\right|^{p}\right) d s \\
& \leq I_{1}+I_{2}+I_{3}+I_{4},
\end{aligned}
$$


where

$$
\begin{aligned}
& I_{1}=\mathbb{E} \int_{0}^{t} p K_{4}\left|x_{\Delta}(s)\right|^{p-2}\left(1+\left|\bar{x}_{\Delta}(s)\right|^{2}\right) d s, \\
& I_{2}=\mathbb{E} \int_{0}^{t} p\left|x_{\Delta}(s)\right|^{p-2}\left|x_{\Delta}(s)-\bar{x}_{\Delta}(s) \| F_{1}\left(\bar{x}_{\Delta}(s)\right)\right| d s, \\
& I_{3}=\mathbb{E} \int_{0}^{t} p\left|x_{\Delta}(s)\right|^{p-2}\left|x_{\Delta}(s)-\bar{x}_{\Delta}(s) \| F_{\Delta}\left(\bar{x}_{\Delta}(s)\right)\right| d s,
\end{aligned}
$$

and

$$
I_{4}=\lambda \mathbb{E}\left(\int_{0}^{t}\left|x_{\Delta}\left(s^{-}\right)+h\left(\bar{x}_{\Delta}\left(s^{-}\right)\right)\right|^{p}-\left|x_{\Delta}\left(s^{-}\right)\right|^{p}\right) d s .
$$

By the Young inequality

$$
a^{p-2} b^{2} \leq \frac{p-2}{p} a^{p}+\frac{2}{p} b^{p}, \quad \forall a, b \geq 0,
$$

we have

$$
I_{1} \leq C\left(1+\int_{0}^{t}\left(\mathbb{E}\left|x_{\Delta}(s)\right|^{p}+\mathbb{E}\left|\bar{x}_{\Delta}(s)\right|^{p}\right) d s\right) .
$$

Similarly, we can show that

$$
I_{2} \leq C\left(1+\int_{0}^{t}\left(\mathbb{E}\left|x_{\Delta}(s)\right|^{p}+\mathbb{E}\left|\bar{x}_{\Delta}(s)\right|^{p}\right) d s\right) .
$$

By Assumption 3.1 it is not difficult to prove that there exists a positive constant $c_{1}$ such that

$$
\left|x_{\Delta}\left(s^{-}\right)+h\left(\bar{x}_{\Delta}\left(s^{-}\right)\right)\right|^{p}-\left|x_{\Delta}\left(s^{-}\right)\right|^{p} \leq c_{1}\left(1+\left|x_{\Delta}\left(s^{-}\right)\right|^{p}+\left|\bar{x}_{\Delta}\left(s^{-}\right)\right|^{p}\right) .
$$

Hence, we have

$$
I_{4} \leq C\left(1+\int_{0}^{t}\left(\mathbb{E}\left|x_{\Delta}(s)\right|^{p}+\mathbb{E}\left|\bar{x}_{\Delta}(s)\right|^{p}\right) d s\right) .
$$

Moreover, the triangle inequality gives

$$
\begin{aligned}
I_{3}= & \mathbb{E} \int_{0}^{t} p\left|x_{\Delta}(s)\right|^{p-2}\left|x_{\Delta}(s)-\bar{x}_{\Delta}(s) \| F_{\Delta}\left(\bar{x}_{\Delta}(s)\right)\right| d s \\
\leq & C \mathbb{E} \int_{0}^{t}\left|\bar{x}_{\Delta}(s)\right|^{p-2}\left|x_{\Delta}(s)-\bar{x}_{\Delta}(s)\right|\left|F_{\Delta}\left(\bar{x}_{\Delta}(s)\right)\right| d s \\
& +C \mathbb{E} \int_{0}^{t}\left|x_{\Delta}(s)-\bar{x}_{\Delta}(s)\right|^{p-2}\left|x_{\Delta}(s)-\bar{x}_{\Delta}(s) \| F_{\Delta}\left(\bar{x}_{\Delta}(s)\right)\right| d s \\
= & : I_{31}+I_{32} .
\end{aligned}
$$


Due to Lemma 3.6 3.12 and $\Delta^{1 / 4} \varphi(\Delta) \leq \hat{h}$, we have

$$
\begin{aligned}
I_{31} & =C \mathbb{E} \int_{0}^{t}\left|\bar{x}_{\Delta}(s)\right|^{p-2}\left|x_{\Delta}(s)-\bar{x}_{\Delta}(s)\right|\left|F_{\Delta}\left(\bar{x}_{\Delta}(s)\right)\right| d s \\
& \leq C \int_{0}^{t} \mathbb{E}\left[\left|\bar{x}_{\Delta}(s)\right|^{p-2}\left|F_{\Delta}\left(\bar{x}_{\Delta}(s)\right)\right| \mathbb{E}\left(\left|x_{\Delta}(s)-\bar{x}_{\Delta}(s)\right| \mid \mathcal{F}_{\kappa(s)}\right)\right] d s \\
& \leq C \varphi(\Delta) \int_{0}^{t} \mathbb{E}\left(\left|\bar{x}_{\Delta}(s)\right|^{p-2}\right) \varphi(\Delta) \Delta^{1 / 2}\left(1+\left|\bar{x}_{\Delta}(s)\right|\right) d s \\
& \leq C(\varphi(\Delta))^{2} \Delta^{1 / 2} \int_{0}^{t}\left(1+\mathbb{E}\left|\bar{x}_{\Delta}(s)\right|^{p-1}\right) d s \\
& \leq C \Delta^{(0.5-2 \varepsilon)} \int_{0}^{t}\left(1+\mathbb{E}\left|\bar{x}_{\Delta}(s)\right|^{p-1}\right) d s \\
& \leq C \int_{0}^{t}\left(1+\mathbb{E}\left|\bar{x}_{\Delta}(s)\right|^{p}\right) d s .
\end{aligned}
$$

By 3.12, we get

$$
\begin{aligned}
I_{32} & =C \mathbb{E} \int_{0}^{t}\left|x_{\Delta}(s)-\bar{x}_{\Delta}(s)\right|^{p-2}\left|x_{\Delta}(s)-\bar{x}_{\Delta}(s) \| F_{\Delta}\left(\bar{x}_{\Delta}(s)\right)\right| d s \\
& \leq C \varphi(\Delta) \int_{0}^{t} \mathbb{E}\left|x_{\Delta}(s)-\bar{x}_{\Delta}(s)\right|^{p-1} d s .
\end{aligned}
$$

It is easy to deduce that when $p \geq 2$, we have

$$
p \varepsilon \leq(p-1) / 2,
$$

for $\varepsilon \in(0,1 / 4]$. This means

$$
\Delta^{(p-1) / 2-p \varepsilon} \leq 1
$$

For $2 \leq p<3$, Lemma 3.6 gives

$$
\mathbb{E}\left(\left|x_{\Delta}(s)-\bar{x}_{\Delta}(s)\right|^{p-1} \mid \mathcal{F}_{\kappa(s)}\right) \leq C \varphi(\Delta)^{p-1} \Delta^{(p-1) / 2}\left(1+\left|\bar{x}_{\Delta}(s)\right|^{p-1}\right) .
$$

Hence, by 3.33, 3.34 and 3.35, we obtain

$$
\begin{aligned}
I_{32} & \leq C(\varphi(\Delta))^{p} \Delta^{(p-1) / 2}\left(1+\mathbb{E}\left|\bar{x}_{\Delta}(s)\right|^{p-1}\right) \\
& \leq C \Delta^{(p-1) / 2-p \varepsilon}\left(1+\mathbb{E}\left|\bar{x}_{\Delta}(s)\right|^{p-1}\right) \\
& \leq C\left(1+\mathbb{E}\left|\bar{x}_{\Delta}(s)\right|^{p}\right) .
\end{aligned}
$$

Similarly, for $p \geq 3$, we have

$$
\mathbb{E}\left(\left|x_{\Delta}(s)-\bar{x}_{\Delta}(s)\right|^{p-1} \mid \mathcal{F}_{\kappa(s)}\right) \leq C\left(\varphi(\Delta)^{p-1} \Delta^{(p-1) / 2}+\Delta\right)\left(1+\left|\bar{x}_{\Delta}(s)\right|^{p-1}\right) .
$$

Substituting this into 3.33 and using 3.34, we also have

$$
\begin{aligned}
I_{32} & \leq C\left((\varphi(\Delta))^{p} \Delta^{(p-1) / 2}+\varphi(\Delta) \Delta\right)\left(1+\mathbb{E}\left|\bar{x}_{\Delta}(s)\right|^{p-1}\right) \\
& \leq C\left(1+\mathbb{E}\left|\bar{x}_{\Delta}(s)\right|^{p}\right) .
\end{aligned}
$$


Hence, by 3.31, 3.32, 3.36 and 3.37, we have

$$
I_{3} \leq C\left(1+\int_{0}^{t} \mathbb{E}\left|\bar{x}_{\Delta}(s)\right|^{p} d s\right) .
$$

Substituting (3.27), (3.28), 3.30) and 3.38) into 3.22), we get

$$
\begin{aligned}
\mathbb{E}\left|x_{\Delta}(t)\right|^{p} & \leq C\left(\int_{0}^{t}\left(1+\mathbb{E}\left|x_{\Delta}(s)\right|^{p}+\mathbb{E}\left|\bar{x}_{\Delta}(s)\right|^{p}\right) d s\right) \\
& \leq C\left(1+\int_{0}^{t} \sup _{0 \leq u \leq s} \mathbb{E}\left|x_{\Delta}(u)\right|^{p} d s\right) .
\end{aligned}
$$

Then, we have

$$
\sup _{0 \leq u \leq t} \mathbb{E}\left|x_{\Delta}(u)\right|^{p} \leq C\left(1+\int_{0}^{t} \sup _{0 \leq u \leq s} \mathbb{E}\left|x_{\Delta}(u)\right|^{p} d s\right) .
$$

The Gronwall inequality yields

$$
\sup _{0 \leq u \leq T} \mathbb{E}\left|x_{\Delta}(u)\right|^{p} \leq C .
$$

75 As this holds for any $\Delta \in(0,1]$ and $C$ is independent of $\Delta$, we obtain the required assertion.

The following lemma shows that $x_{\Delta}(t)$ and $\bar{x}_{\Delta}(t)$ are close to each other in the sense of $\mathcal{L}^{p}$.

Lemma 3.8. Let Assumption 3.1 and 3.3 hold. Then, for all $\Delta \in(0,1]$ and $t \in(0, T]$,

$$
\begin{aligned}
& \mathbb{E}\left|x_{\Delta}(t)-\bar{x}_{\Delta}(t)\right|^{p} \leq C\left((\varphi(\Delta))^{p} \Delta^{p / 2}+\Delta\right), \quad 2 \leq p<\bar{p}, \\
& \mathbb{E}\left|x_{\Delta}(t)-\bar{x}_{\Delta}(t)\right|^{p} \leq C(\varphi(\Delta))^{p} \Delta^{p / 2}, \quad 0<p<2 .
\end{aligned}
$$

Consequently, for any $p>0$,

$$
\lim _{\Delta \rightarrow 0} \mathbb{E}\left|x_{\Delta}(t)-\bar{x}_{\Delta}(t)\right|^{p}=0 .
$$

Proof. For any $p \geq 2$, by Lemma 3.7, we have

$$
\sup _{0 \leq \Delta \leq 1} \sup _{0 \leq t \leq T} \mathbb{E}\left|x_{\Delta}(t)\right|^{p} \leq C .
$$

Inserting (3.42) into 3.17) gives 3.39]. For any $p \in(0,2)$, the Hölder inequality implies

$$
\begin{aligned}
& \mathbb{E}\left|x_{\Delta}(t)-\bar{x}_{\Delta}(t)\right|^{p} \leq\left(\mathbb{E}\left|x_{\Delta}(t)-\bar{x}_{\Delta}(t)\right|^{2}\right)^{p / 2} \\
& \leq C\left((\varphi(\Delta))^{2} \Delta+\Delta\right)^{p / 2} \leq C\left((\varphi(\Delta))^{p} \Delta^{p / 2}+\Delta^{p / 2}\right) \leq C(\varphi(\Delta))^{p} \Delta^{p / 2} .
\end{aligned}
$$

Thus, we obtain (3.41) from (3.39) and (3.40).

Let us propose two lemmas before we state our main results in this paper.

Lemma 3.9. Let Assumption 3.1 and 3.3 hold. For any real number $n>\left|x_{0}\right|$, define the stopping time

$$
\tau_{n}=\inf \{t \geq 0:|x(t)| \geq n\} .
$$

Then

$$
\mathbb{P}\left(\tau_{n} \leq T\right) \leq \frac{C}{n^{2}} .
$$


Proof. The proof is given in the Appendix.

Lemma 3.10. Let Assumption 3.1 and 3.3 hold. For any real number $n>\left|x_{0}\right|$, define the stopping time,

$$
\rho_{\Delta, n}=\inf \left\{t \geq 0:\left|x_{\Delta}(t)\right| \geq n\right\}
$$

Then

$$
\mathbb{P}\left(\rho_{\Delta, n} \leq T\right) \leq \frac{C}{n^{2}}
$$

80 Proof. The proof is given in the Appendix.

Now, we show one of our main results in our paper. The proof is similar to that of Theorem 3.6 in [28], we only highlight the different parts.

Theorem 3.11. Let Assumption 3.1, 3.2 and 3.3 hold and assume that there exists a number $p \in(2, \bar{p})$ such that

$$
p>(1+\gamma) \bar{r}
$$

Let $r \in[2, \bar{r})$ be arbitrary. Then for any $\Delta \in(0,1]$,

$$
\mathbb{E}\left|x(T)-x_{\Delta}(T)\right|^{r} \leq C\left(\left(\mu^{-1}(\varphi(\Delta))\right)^{-(p-(1+\gamma) r)}+(\varphi(\Delta))^{r} \Delta^{r / 2}+\Delta^{(p-\gamma r) / p}\right)
$$

and

$$
\mathbb{E}\left|x(T)-\bar{x}_{\Delta}(T)\right|^{r} \leq C\left(\left(\mu^{-1}(\varphi(\Delta))\right)^{-(p-(1+\gamma) r)}+(\varphi(\Delta))^{r} \Delta^{r / 2}+\Delta^{(p-\gamma r) / p}\right) .
$$

In particular, we define

$$
\mu(n)=L_{4} n^{1+\gamma}, \quad n \geq 1
$$

with $L_{4}=2 L_{1}+|F(0)|+|G(0)|$ and let

$$
\varphi(\Delta)=\hat{h} \Delta^{-\varepsilon}, \quad \text { for some } \quad \varepsilon \in(0,1 / 4]
$$

to obtain

$$
\mathbb{E}\left|x(T)-x_{\Delta}(T)\right|^{r} \leq C \Delta^{[\varepsilon(p-(1+\gamma) r) /(1+\gamma)] \wedge[r(1-2 \varepsilon) / 2] \wedge[(p-\gamma r) / p]}
$$

and

$$
\mathbb{E}\left|x(T)-\bar{x}_{\Delta}(T)\right|^{r} \leq C \Delta^{[\varepsilon(p-(1+\gamma) r) /(1+\gamma)] \wedge[r(1-2 \varepsilon) / 2] \wedge[(p-\gamma r) / p]}
$$

for all $\Delta \in(0,1]$.

Proof. Let $\Delta \in(0,1]$ be arbitrary. Let $e_{\Delta}(t)=x(t)-x_{\Delta}(t)$ for $t>0$. Fix a number $q \in(r, \bar{r})$, 3.45 means $p>(1+\gamma) q$. For any integer $n>\left|x_{0}\right|$, define the stopping time

$$
\sigma_{n}=\inf \left\{t \geq 0:|x(t)| \vee\left|x_{\Delta}(t)\right| \geq n\right\} .
$$


By the Itô formula, we get that for $0 \leq t \leq T$

$$
\begin{aligned}
& \mathbb{E}\left|e_{\Delta}\left(t \wedge \sigma_{n}\right)\right|^{r} \\
& \leq \mathbb{E} \int_{0}^{t \wedge \sigma_{n}} r\left|e_{\Delta}(s)\right|^{r-2}\left(e_{\Delta}^{T}(s)\left(f(x(s))-f_{\Delta}\left(\bar{x}_{\Delta}(s)\right)\right)+\frac{r-1}{2}\left|g(x(s))-g_{\Delta}\left(\bar{x}_{\Delta}(s)\right)\right|^{2}\right) d s \\
& \quad+\lambda \mathbb{E} \int_{0}^{t \wedge \sigma_{n}}\left(\left|e_{\Delta}(s)+\left(h\left(x\left(s^{-}\right)\right)-h\left(\bar{x}_{\Delta}\left(s^{-}\right)\right)\right)\right|^{r}-\left|e_{\Delta}(s)\right|^{r}\right) d s \\
& =: J_{1}+J_{2} .
\end{aligned}
$$

Let us estimate $J_{2}$ first. Using Assumption 3.1 gives

$$
\begin{aligned}
& \left|x\left(s^{-}\right)-x_{\Delta}(s)+h\left(x\left(s^{-}\right)\right)-h\left(\bar{x}_{\Delta}(s)\right)\right|^{r} \\
& \leq 2^{r-1}\left(\left|x\left(s^{-}\right)-x_{\Delta}(s)\right|^{r}+\left|h\left(x\left(s^{-}\right)\right)-h\left(\bar{x}_{\Delta}(s)\right)\right|^{r}\right) \\
& \leq 2^{r-1}\left(\left|x\left(s^{-}\right)-x_{\Delta}(s)\right|^{r}+L_{1}^{r}\left|x\left(s^{-}\right)-\bar{x}_{\Delta}(s)\right|^{r}\right) \\
& \leq c_{2}\left(\left|x\left(s^{-}\right)-x_{\Delta}(s)\right|^{r}+\left|x_{\Delta}(s)-\bar{x}_{\Delta}(s)\right|^{r}\right),
\end{aligned}
$$

where $c_{2}=2^{r-1}\left(1+L_{1}^{r} 2^{r-1}\right)>1$. Hence, by Lemma 3.8 , we have

$$
\begin{aligned}
J_{2} & \leq \lambda\left(c_{2}-1\right) \int_{0}^{t} \mathbb{E}\left|e_{\Delta}\left(s \wedge \sigma_{n}\right)\right|^{r} d s+\lambda c_{2} \int_{0}^{T} \mathbb{E}\left|x_{\Delta}(s)-\bar{x}_{\Delta}(s)\right|^{r} d s \\
& \leq \lambda\left(c_{2}-1\right) \int_{0}^{t} \mathbb{E}\left|e_{\Delta}\left(s \wedge \sigma_{n}\right)\right|^{r} d s+C\left(\Delta^{r / 2}(\varphi(\Delta))^{r}+\Delta\right) .
\end{aligned}
$$

By the elementary inequalty, $J_{1}$ can be decomposed into two parts denoted by $J_{1}=J_{3}+J_{4}$, where

$$
\begin{aligned}
J_{3}=\mathbb{E} \int_{0}^{t \wedge \sigma_{n}} r\left|e_{\Delta}(s)\right|^{r-2} & \left(e_{\Delta}^{T}(s)\left(f(x(s))-f\left(x_{\Delta}(s)\right)\right)\right. \\
& \left.+\frac{q-1}{2}\left|g(x(s))-g\left(x_{\Delta}(s)\right)\right|^{2}\right) d s
\end{aligned}
$$

and

$$
\begin{aligned}
J_{4}=\mathbb{E} \int_{0}^{t \wedge \sigma_{n}} r\left|e_{\Delta}(s)\right|^{r-2} & \left(e_{\Delta}^{T}(s)\left(f\left(x_{\Delta}(s)\right)\right)-f_{\Delta}\left(\bar{x}_{\Delta}(s)\right)\right) \\
& \left.+\frac{(r-1)(q-1)}{2(q-r)}\left|g\left(x_{\Delta}(s)\right)-g_{\Delta}\left(\bar{x}_{\Delta}(s)\right)\right|^{2}\right) d s .
\end{aligned}
$$

By 3.6 , we have

$$
J_{3} \leq r L_{3} \int_{0}^{t \wedge \sigma_{n}} \mathbb{E}\left|e_{\Delta}(s)\right|^{r} d s
$$


The elementary inequality gives

$$
\begin{aligned}
J_{4} \leq & \mathbb{E} \int_{0}^{t \wedge \sigma_{n}} r\left|e_{\Delta}(s)\right|^{r-2}\left(e_{\Delta}^{T}(s)\left(f\left(x_{\Delta}(s)\right)\right)-f_{\Delta}\left(x_{\Delta}(s)\right)\right) \\
& \left.+\frac{(r-1)(q-1)}{(q-r)}\left|g\left(x_{\Delta}(s)\right)-g_{\Delta}\left(x_{\Delta}(s)\right)\right|^{2}\right) d s \\
+ & \mathbb{E} \int_{0}^{t \wedge \sigma_{n}} r\left|e_{\Delta}(s)\right|^{r-2}\left(e_{\Delta}^{T}(s)\left(f_{\Delta}\left(x_{\Delta}(s)\right)\right)-f_{\Delta}\left(\bar{x}_{\Delta}(s)\right)\right) \\
& \left.+\frac{(r-1)(q-1)}{(q-r)}\left|g_{\Delta}\left(x_{\Delta}(s)\right)-g_{\Delta}\left(\bar{x}_{\Delta}(s)\right)\right|^{2}\right) d s \\
= & : J_{41}+J_{42} .
\end{aligned}
$$

In the same way as Theorem 3.6 in [28] was proved, we can show that

$$
J_{41} \leq C\left(\int_{0}^{t \wedge \sigma_{n}} \mathbb{E}\left|e_{\Delta}(s)\right|^{r} d s+\left(\mu^{-1}(\varphi(\Delta))\right)^{-(p-(1+\gamma) r)}\right)
$$

and

$$
\begin{aligned}
J_{42} & \leq C \int_{0}^{t \wedge \sigma_{n}} \mathbb{E}\left|e_{\Delta}(s)\right|^{r} d s+C \int_{0}^{T}\left(\mathbb{E}\left|x_{\Delta}(s)-\bar{x}_{\Delta}(s)\right|^{p r /(p-\gamma r)}\right)^{(p-\gamma r) / p} \\
& \leq C \int_{0}^{t \wedge \sigma_{n}} \mathbb{E}\left|e_{\Delta}(s)\right|^{r} d s+C\left((\varphi(\Delta))^{p r /(p-\gamma r)} \Delta^{0.5 p r /(p-\gamma r)}+\Delta\right)^{(p-\gamma r) / p} \\
& \leq C \int_{0}^{t \wedge \sigma_{n}} \mathbb{E}\left|e_{\Delta}(s)\right|^{r} d s+C\left((\varphi(\Delta))^{r} \Delta^{r / 2}+\Delta^{(p-\gamma r) / p}\right),
\end{aligned}
$$

where we use Lemma 3.8 and the fact that

$$
\frac{p r}{p-\gamma r}=r \frac{p}{p-\gamma r}>2 .
$$

Inserting (3.58) and 3.59) into 3.57), we have

$$
J_{4} \leq C\left(\int_{0}^{t} \mathbb{E}\left|e_{\Delta}\left(s \wedge \sigma_{n}\right)\right|^{r} d s+\left(\mu^{-1}(\varphi(\Delta))\right)^{-(p-(1+\gamma) r)}+(\varphi(\Delta))^{r} \Delta^{r / 2}+\Delta^{(p-\gamma r) / p}\right) .
$$

Combing (3.53), 3.56) and 3.60, we have

$$
\begin{aligned}
\mathbb{E}\left|e_{\Delta}\left(t \wedge \sigma_{n}\right)\right|^{r} \leq & C\left(\int_{0}^{t} \mathbb{E}\left|e_{\Delta}\left(s \wedge \sigma_{n}\right)\right|^{r} d s+\left(\mu^{-1}(\varphi(\Delta))\right)^{-(p-(1+\gamma) r)}\right. \\
& \left.+(\varphi(\Delta))^{r} \Delta^{r / 2}+\Delta^{(p-\gamma r) / p}\right)
\end{aligned}
$$

The Gronwall inequality implies

$$
\mathbb{E}\left|e_{\Delta}\left(T \wedge \sigma_{n}\right)\right|^{r} \leq C\left(\left(\mu^{-1}(\varphi(\Delta))\right)^{-(p-(1+\gamma) r)}+(\varphi(\Delta))^{r} \Delta^{r / 2}+\Delta^{(p-\gamma r) / p}\right) .
$$

Using Lemma 3.9 and 3.10 and letting $n \rightarrow \infty$ gives the desired assertion 3.46. By (3.46) 85 and Lemma 3.8 gives the another assertion (3.47). Recalling (3.48), then $\mu^{-1}(x)=\left(x / L_{4}\right)^{1 /(1+\gamma)}$. Substituting this and (3.49) into (3.46) gives 3.50). Similarly, we can get (3.51). Thus, the proof is complete.

The following corollary reveals the optimal $\mathcal{L}^{r}$-convergence rate of truncated EM method. 
Corollary 3.12. Let Assumption 3.1, 3.2 hold and suppose that Assumption 3.3 holds for all $\bar{p} \in(\bar{r}, \infty)$. Let $\mu$ and $\varphi$ be defined in (3.48) and 3.49). Then, for any

$$
\begin{gathered}
r \in[2, \bar{r}), \quad p \in((1+\gamma) r \vee \bar{r}, \bar{p}) \quad \text { and } \quad \varepsilon \in(0,1 / 4], \\
\mathbb{E}\left|x(T)-x_{\Delta}(T)\right|^{r} \leq C \Delta^{[r(1-2 \varepsilon) / 2] \wedge[(p-\gamma r) / p]}
\end{gathered}
$$

and

$$
\mathbb{E}\left|x(T)-\bar{x}_{\Delta}(T)\right|^{r} \leq C \Delta^{[r(1-2 \varepsilon) / 2] \wedge[(p-\gamma r) / p]} .
$$

Proof. We choose $p$ sufficiently large such that

$$
p \geq \frac{(1+\gamma) r}{2 \varepsilon},
$$

which means

$$
\varepsilon(p-(1+\gamma) r) /(1+\gamma) \geq r(1-2 \varepsilon) / 2 .
$$

By (3.50) and 3.51, we obtain 3.62) and 3.63.

$90 \quad$ Remark 3.13. Replacing condition (3.45), that is $p>(1+\gamma) \bar{r}$, by a weaker one $p>(1+\gamma) r \vee \bar{r}$ does not affect the results in Theorem 3.11] But, this small change will make the choice of p more flexible in simulation.

Remark 3.14. The Corollary 3.12 shows that the order of $\mathcal{L}^{r}$-convergence of truncated EM method for $S D E$ [2.1], namely $[r(1-2 \varepsilon) / 2] \wedge[(p-\gamma r) / p]$, is close to 1 . This is almost optimal $\mathcal{L}^{r}$-convergence rate, if we recall that under the global Lipschitz condition the classical EM method has order 1 of $\mathcal{L}^{r}$-convergence. It should be mentioned that this is significantly different from the result on SDEs without jumps. We already known that for any $r \geq 2$ (see [28])

$$
\mathbb{E}\left|x(T)-x_{\Delta}(T)\right|^{r} \leq C \Delta^{r(1-2 \varepsilon) / 2},
$$

which means that the $\mathcal{L}^{r}$-convergence order is close to r/2 when there is no jumps in SDE 2.1].

95 In fact, this difference is caused by the following reason: all moments of the Poisson increments $\Delta N_{k}=N((k+1) \Delta)-N(k \Delta)$ have the same order $\Delta$ (see (3.20)), while the Brownian increments $\Delta B_{k}=B((k+1) \Delta)-B(k \Delta)$ have different orders, namely $\mathbb{E}\left|\Delta B_{k}\right|^{2 n}=o\left(\Delta^{n}\right)$ and $\mathbb{E}\left|\Delta B_{k}\right|^{2 n+1}=0$. These properties eventually lead to the differences in the convergence order between SDEs with and without jumps.

3.2. Convergence and convergence order of the truncated EM method in $\mathcal{L}^{r}(0<r<2)$

In this subsection, we discuss the convergence and convergence rate in $\mathcal{L}^{r}(0<r<2)$ under the assumption that the drift, diffusion and jump terms behave like a polynomial. For this purpose, we first impose the following assumptions.

Assumption 3.15. There exists a positive constant $K_{n}$ such that

$$
|f(x)-f(y)| \vee|g(x)-g(y)| \vee|h(x)-h(y)| \leq K_{n}|x-y|, \quad \forall x, y \in \mathbb{R}^{d},|x| \vee|y| \leq n .
$$


Assumption 3.16. There exists a constant $\bar{K}>0$ such that

$$
2 x^{T} f(x)+|g(x)|^{2}+\lambda\left(2 x^{T} h(x)+|h(x)|^{2}\right) \leq \bar{K}\left(1+|x|^{2}\right), \quad \forall x \in \mathbb{R}^{d} .
$$

We also give a known result as a lemma (see [7]).

Lemma 3.17. Under Assumption 3.15 and 3.16, the SDE [2.1] has a unique global solution $x(t)$, moreover,

$$
\sup _{0 \leq t \leq T} \mathbb{E}|x(t)|^{2}<\infty, \quad \forall T>0 .
$$

In this subsection, all the three coefficients of the SDE are allowed to grow super-linearly. Hence, we have to truncate the three coefficients. Similarly, we first choose a strictly increasing function $\mu: \mathbb{R}^{+} \rightarrow \mathbb{R}^{+}$such that $\mu(n) \rightarrow \infty$, as $n \rightarrow \infty$, and

$$
\sup _{|x| \leq n}|f(x)| \vee|g(x)| \vee|h(x)| \leq \mu(n), \quad \forall n \geq 1 .
$$

The inverse function of $\mu$ is denoted by $\mu^{-1}$. We choose a strictly decreasing function $\varphi:(0,1] \rightarrow$ $(0, \infty)$ such that

$$
\lim _{\Delta \rightarrow 0} \varphi(\Delta)=\infty \quad \text { and } \quad \varphi(\Delta) \Delta^{1 / 4} \leq 1, \quad \forall \Delta \in(0,1]
$$

For a given step size $\Delta \in(0,1]$, the truncated functions are defined as below

$$
f_{\Delta}(x)=f\left(\pi_{\Delta}(x)\right), \quad g_{\Delta}(x)=g\left(\pi_{\Delta}(x)\right) \quad \text { and } \quad h_{\Delta}(x)=h\left(\pi_{\Delta}(x)\right), \quad \forall x \in \mathbb{R}^{d},
$$

where $\pi_{\Delta}$ is defined as the same as before. It is useful to note that

$$
\left|f_{\Delta}(x)\right| \vee\left|g_{\Delta}(x)\right| \vee\left|h_{\Delta}(x)\right| \leq \varphi(\Delta), \quad \forall x \in \mathbb{R}^{d} .
$$

The following lemma also shows that the truncated functions preserve the Khaminskii-type condition. The proof is given in the Appendix.

Lemma 3.18. Let Assumption 3.16 hold. Then, for all $\Delta \in(0,1]$,

$$
2 x^{T} f_{\Delta}(x)+\left|g_{\Delta}(x)\right|^{2}+\lambda\left(2 x^{T} h_{\Delta}(x)+\left|h_{\Delta}(x)\right|^{2}\right) \leq 2 \hat{K}\left(1+|x|^{2}\right), \quad \forall x \in \mathbb{R}^{d}
$$

where $\hat{K}=\bar{K}\left[1 \wedge 1 / \mu^{-1}(\varphi(1))\right]$.

Let $M, X_{\Delta}(0), \Delta B_{k}, \Delta N_{k}$ and $\bar{x}_{\Delta}(t)$ be the same as before. We now define the discrete-time truncated EM scheme

$$
X_{\Delta}\left(t_{k+1}\right)=X_{\Delta}\left(t_{k}\right)+f_{\Delta}\left(X_{\Delta}\left(t_{k}\right)\right) \Delta+g_{\Delta}\left(X_{\Delta}\left(t_{k}\right)\right) \Delta B_{k}+h_{\Delta}\left(X_{\Delta}\left(t_{k}^{-}\right)\right) \Delta N_{k}, \quad 0 \leq k \leq M-1 .
$$

The continuous-time form is defined by

$$
x_{\Delta}(t)=x_{0}+\int_{0}^{t} f_{\Delta}\left(\bar{x}_{\Delta}(s)\right) d s+\int_{0}^{t} g_{\Delta}\left(\bar{x}_{\Delta}(s)\right) d B(s)+\int_{0}^{t} h_{\Delta}\left(\bar{x}_{\Delta}\left(s^{-}\right)\right) d N(s) .
$$

In order to state our main results, we first give some useful lemmas. 
Lemma 3.19. For any $\Delta \in(0,1]$ and $t>0$. Then

$$
\begin{aligned}
& \mathbb{E}\left|x_{\Delta}(t)-\bar{x}_{\Delta}(t)\right|^{\hat{p}} \leq C_{\hat{p}}(\varphi(\Delta))^{\hat{p}} \Delta, \quad \hat{p} \geq 2, \\
& \mathbb{E}\left|x_{\Delta}(t)-\bar{x}_{\Delta}(t)\right|^{\hat{p}} \leq C_{\hat{p}}(\varphi(\Delta))^{\hat{p}} \Delta^{\hat{p} / 2}, \quad 0<\hat{p}<2 .
\end{aligned}
$$

Consequently,

$$
\lim _{\Delta \rightarrow 0} \mathbb{E}\left|x_{\Delta}(t)-\bar{x}_{\Delta}(t)\right|^{\hat{p}}=0, \quad \forall t \geq 0 .
$$

Proof. Fix any $\Delta \in(0,1], t \geq 0$ and $\hat{p} \geq 2$. There is an integer $k \geq 0$ such that $t_{k} \leq t<t_{k+1}$. By Assumption 3.1 and 3.70 , we have

$$
\begin{aligned}
& \mathbb{E}\left|x_{\Delta}(t)-\bar{x}_{\Delta}(t)\right|^{\hat{p}} \\
& \leq C_{\hat{p}}\left(\mathbb{E}\left|\int_{t_{k}}^{t} f_{\Delta}\left(\bar{x}_{\Delta}(s)\right) d s\right|^{\hat{p}}+\left.\mathbb{E}\left|\int_{t_{k}}^{t} g_{\Delta}\left(\bar{x}_{\Delta}(s)\right) d B(s)\right|\right|^{\hat{p}}+\mathbb{E}\left|\int_{t_{k}}^{t} h_{\Delta}\left(\bar{x}_{\Delta}\left(s^{-}\right)\right) d N(s)\right|^{\hat{p}}\right) \\
& \leq C_{\hat{p}}\left(\Delta^{\hat{p}-1} \mathbb{E} \int_{t_{k}}^{t}\left|f_{\Delta}\left(\bar{x}_{\Delta}(s)\right)\right|^{\hat{p}} d s+\Delta^{(\hat{p}-2) / 2} \mathbb{E} \int_{t_{k}}^{t}\left|g_{\Delta}\left(\bar{x}_{\Delta}(s)\right)\right|^{\hat{p}} d s+\mathbb{E}\left|\int_{t_{k}}^{t} h_{\Delta}\left(\bar{x}_{\Delta}\left(s^{-}\right)\right) d N(s)\right|^{\hat{p}}\right) \\
& \leq C_{\hat{p}}\left(\Delta^{p / 2}(\varphi(\Delta))^{\hat{p}}+\mathbb{E}\left|\int_{t_{k}}^{t} h_{\Delta}\left(\bar{x}_{\Delta}\left(s^{-}\right)\right) d N(s)\right|^{\hat{p}}\right)
\end{aligned}
$$

where $C_{\hat{p}}$ is a generic constant. The property of Poisson increments implies

$$
\begin{aligned}
\mathbb{E}\left|\int_{t_{k}}^{t} h_{\Delta}\left(\bar{x}_{\Delta}\left(s^{-}\right)\right) d N(s)\right|^{\hat{p}} & \leq(\varphi(\Delta))^{\hat{p}} \mathbb{E}\left|\Delta N_{k}\right|^{\hat{p}} \\
& \leq c_{0}(\varphi(\Delta))^{\hat{p}} \Delta .
\end{aligned}
$$

Inserting this into 3.77) and recalling $\hat{p} \geq 2$ gives

$$
\mathbb{E}\left|x_{\Delta}(t)-\bar{x}_{\Delta}(t)\right|^{\hat{p}} \leq C_{\hat{p}}(\varphi(\Delta))^{\hat{p}} \Delta .
$$

Noting from 3.10 that $(\varphi(\Delta))^{\hat{p}} \Delta=(\varphi(\Delta))^{\hat{p}} \Delta^{1 / 2} \Delta^{1 / 2} \leq \Delta^{1 / 2}$, we obtain 3.76 form 3.74).

For $0<\hat{p}<2$, we have

$$
\begin{aligned}
& \mathbb{E}\left|x_{\Delta}(t)-\bar{x}_{\Delta}(t)\right|^{\hat{p}} \leq\left(\mathbb{E}\left|x_{\Delta}(t)-\bar{x}_{\Delta}(t)\right|^{2}\right)^{\hat{p} / 2} \\
& \leq\left(C_{\hat{p}}(\varphi(\Delta))^{2} \Delta\right)^{\hat{p} / 2}=C_{\hat{p}}(\varphi(\Delta))^{\hat{p}} \Delta^{\hat{p} / 2}
\end{aligned}
$$

Thus, the proof is complete.

The following lemma reveals the boundedness of the second moments for the truncated EM solutions.

Lemma 3.20. Let Assumption 3.15 and 3.16 hold. Then

$$
\sup _{0 \leq \Delta \leq 1} \sup _{0 \leq t \leq T} \mathbb{E}\left|x_{\Delta}(t)\right|^{2} \leq C, \quad \forall T>0 .
$$


Proof. Fix any $\Delta \in(0,1]$ and $T>0$. By the Itô formula and Assumption 3.16, we have

$$
\begin{aligned}
\mathbb{E}\left|x_{\Delta}(t)\right|^{2} \leq & \mathbb{E}\left|x_{0}\right|^{2}+\mathbb{E} \int_{0}^{t}\left(2 x_{\Delta}^{T}(s) f_{\Delta}\left(\bar{x}_{\Delta}(s)\right)+\left|g_{\Delta}\left(\bar{x}_{\Delta}(s)\right)\right|^{2}\right) d s \\
& +\lambda \mathbb{E} \int_{0}^{t}\left(2 x_{\Delta}(s)^{T} h_{\Delta}\left(\bar{x}_{\Delta}\left(s^{-}\right)\right)+\left|h_{\Delta}\left(\bar{x}_{\Delta}\left(s^{-}\right)\right)\right|^{2}\right) d s \\
\leq & \mathbb{E}\left|x_{0}\right|^{2}+\mathbb{E} \int_{0}^{t}\left(2 \bar{x}_{\Delta}^{T}(s) f_{\Delta}\left(\bar{x}_{\Delta}(s)\right)+\left|g_{\Delta}\left(\bar{x}_{\Delta}(s)\right)\right|^{2}\right) d s \\
& +\lambda \mathbb{E} \int_{0}^{t}\left(2 \bar{x}_{\Delta}^{T}(s) h_{\Delta}\left(\bar{x}_{\Delta}\left(s^{-}\right)\right)+\left|h_{\Delta}\left(\bar{x}_{\Delta}\left(s^{-}\right)\right)\right|^{2}\right) d s+\bar{J}_{1} \\
\leq & \mathbb{E}\left|x_{0}\right|^{2}+2 \hat{K} \int_{0}^{t}\left(1+\mathbb{E}\left|\bar{x}_{\Delta}(s)\right|^{2}\right) d s+\bar{J}_{1},
\end{aligned}
$$

where

$$
\bar{J}_{1}=\mathbb{E} \int_{0}^{t}\left(2\left(x_{\Delta}(s)-\bar{x}_{\Delta}(s)\right)^{T} f_{\Delta}\left(\bar{x}_{\Delta}(s)\right)+2 \lambda\left(x_{\Delta}(s)-\bar{x}_{\Delta}(s)\right)^{T} h_{\Delta}\left(\bar{x}_{\Delta}\left(s^{-}\right)\right)\right) d s .
$$

By Lemma 3.19, 3.68 and 3.69, we have

$$
\begin{aligned}
\bar{J}_{1} & \leq 2(\lambda+1) \varphi(\Delta) \int_{0}^{t} \mathbb{E}\left|x_{\Delta}(s)-\bar{x}_{\Delta}(s)\right| d s \\
& \leq 2(\lambda+1) T C(\varphi(\Delta))^{2} \Delta^{1 / 2} \leq C
\end{aligned}
$$

Inserting this into 3.79) and using Lemma 3.19 gives

$$
\mathbb{E}\left|x_{\Delta}(t)\right|^{2} \leq C+2 \bar{K} \int_{0}^{t} \mathbb{E}\left|\bar{x}_{\Delta}(s)\right|^{2} d s .
$$

Hence, we have

$$
\sup _{0 \leq u \leq t} \mathbb{E}\left|x_{\Delta}(u)\right|^{2} \leq C+2 \bar{K} \int_{0}^{t} \sup _{0 \leq u \leq s} \mathbb{E}\left|x_{\Delta}(u)\right|^{2} d s .
$$

The Gronwall inequality yields

$$
\sup _{0 \leq u \leq T} \mathbb{E}\left|x_{\Delta}(u)\right|^{2} \leq C
$$

Thus, we complete the proof.

As the proof is in a similar way as Lemma 3.10 and 3.11 were proved, we also have the following Lemma.

Lemma 3.21. Let Assumption 3.15 and 3.16 hold. For any real number $n>\left|x_{0}\right|$, then

$$
\mathbb{P}\left(\tau_{n} \leq T\right) \leq \frac{C}{n^{2}} \quad \text { and } \quad \mathbb{P}\left(\rho_{\Delta, n} \leq T\right) \leq \frac{C}{n^{2}},
$$

where $\tau_{n}$ and $\rho_{\Delta, n}$ is the same as before.

Now, let us discuss the convergence of the truncated EM method for SDEs with Poisson jumps. 
Theorem 3.22. Let Assumption 3.15 and 3.16 hold. Then, for any $r \in(0,2)$

$$
\lim _{\Delta \rightarrow 0} \mathbb{E}\left|x(T)-x_{\Delta}(T)\right|^{r}=0
$$

and

$$
\lim _{\Delta \rightarrow 0} \mathbb{E}\left|x(T)-\bar{x}_{\Delta}(T)\right|^{r}=0 .
$$

Proof. Let $\tau_{n}, \rho_{\Delta, n}$, and $e_{\Delta}(t)$ be the same as before. We set $\theta_{\Delta, n}=\tau_{n} \wedge \rho_{\Delta, n}$. Applying the Young inequality, we have that for any $\delta>0$,

$$
\begin{aligned}
\mathbb{E}\left|e_{\Delta}(T)\right|^{r} & =\mathbb{E}\left(\left|e_{\Delta}(T)\right|^{r} \mathbb{I}_{\left\{\theta_{\Delta, n}>T\right\}}\right)+\mathbb{E}\left(\left|e_{\Delta}(T)\right|^{r} \mathbb{I}_{\left\{\theta_{\Delta, n} \leq T\right\}}\right) \\
& \leq \mathbb{E}\left(\left|e_{\Delta}\left(T \wedge \theta_{\Delta, n}\right)\right|^{r}\right)+\frac{r \delta}{2} \mathbb{E}\left|e_{\Delta}(T)\right|^{2}+\frac{2-r}{2 \delta^{r /(2-r)}} \mathbb{P}\left(\theta_{\Delta, n} \leq T\right) .
\end{aligned}
$$

By Lemma 3.17 and 3.20, we have

$$
\mathbb{E}\left|e_{\Delta}(T)\right|^{2} \leq 2 \mathbb{E}|x(T)|^{p}+2 \mathbb{E}\left|x_{\Delta}(T)\right|^{p} \leq C .
$$

Using Lemma 3.21 we obtain

$$
\mathbb{P}\left(\theta_{\Delta, n} \leq T\right) \leq \mathbb{P}\left(\tau_{n} \leq T\right)+\mathbb{P}\left(\rho_{\Delta, n} \leq T\right) \leq \frac{C}{n^{2}} .
$$

Inserting 3.84 and 3.85) into 3.83, we get

$$
\mathbb{E}\left|e_{\Delta}(T)\right|^{r} \leq \mathbb{E}\left|e_{\Delta}\left(T \wedge \theta_{\Delta, n}\right)\right|^{r}+\frac{C r \delta}{2}+\frac{C(2-r)}{2 n^{2} \delta^{r /(2-r)}} .
$$

Now, let $\varepsilon>0$ be arbitrary. We can choose $\delta$ sufficiently small such that

$$
\frac{C r \delta}{2} \leq \frac{\varepsilon}{3}
$$

and then choose $n$ sufficiently large such that

$$
\frac{C(2-r)}{2 n^{2} \delta^{r /(2-r)}} \leq \frac{\varepsilon}{3} .
$$

We may assume that $\Delta^{*}$ is sufficiently small for $\mu^{-1}\left(\varphi\left(\Delta^{*}\right)\right) \geq n$. In the same way as Theorem 3.5 in [14] was proved, we can show that for all $\Delta \in\left(0, \Delta^{*}\right]$

$$
\mathbb{E}\left|e_{\Delta}(T)\right|^{2} \leq C \Delta
$$

which implies

$$
\mathbb{E}\left(\left|e_{\Delta}\left(T \wedge \theta_{\Delta, n}\right)\right|^{r}\right) \leq \frac{\varepsilon}{3} .
$$

Hence, we obtain the required assertion 3.81). Combining this with Lemma 3.19) gives 3.82. Thus, the proof is complete.

For the purpose of getting the convergence order at time $\mathrm{T}$, we need some additional conditions. 
Assumption 3.23. There exists a constant $\bar{L}_{1}>0$ such that

$$
\begin{aligned}
& 2(x-y)^{T}(f(x)-f(y))+|g(x)-g(y)|^{2} \\
& +2 \lambda(x-y)^{T}(h(x)-h(y))+\lambda|h(x)-h(y)|^{2} \leq \bar{L}_{1}|x-y|^{2},
\end{aligned}
$$

for any $x, y \in \mathbb{R}^{d}$.

Assumption 3.24. There exist constant $\bar{L}_{2}>0$ and $0 \leq \bar{\gamma}<1$ such that

$$
|f(x)-f(y)| \vee|h(x)-h(y)| \leq \bar{L}_{2}\left(1+|x|^{\bar{\gamma}}+|y|^{\bar{\gamma}}\right)|x-y|, \quad \forall x, y \in \mathbb{R}^{d} .
$$

Obviously, this condition implies

$$
|f(x)| \vee|h(x)| \leq \bar{L}_{3}|x|^{1+\bar{\gamma}},
$$

where $\bar{L}_{3}=2 \bar{L}_{2}+|f(0)|+|h(0)|$.

Lemma 3.25. Let Assumption 3.15, 3.16, 3.23 and 3.24 hold. Let $n>\left|x_{0}\right|$ be a real number , $\tau_{n}$ and $\rho_{\Delta, n}$ be the same as before. Set

$$
\theta_{\Delta, n}=\tau_{n} \wedge \rho_{\Delta, n} \quad \text { and } \quad e_{\Delta}(t)=x(t)-x_{\Delta}(t), \quad \forall t>0 .
$$

Assume that $\Delta \in(0,1]$ is sufficiently small such that $\mu^{-1}(\varphi(\Delta)) \geq n$. Then

$$
\mathbb{E}\left|e_{\Delta}\left(T \wedge \theta_{\Delta, n}\right)\right|^{2} \leq C(\varphi(\Delta))^{2} \Delta .
$$

Proof. We write $\theta_{\Delta, n}=\theta$ for simplicity. By the Itô formula and Assumption 3.23, we get that for $0 \leq t \leq T$,

$$
\begin{aligned}
& \mathbb{E}\left|e_{\Delta}(t \wedge \theta)\right|^{2} \\
& \leq \mathbb{E} \int_{0}^{t \wedge \theta}\left(2 e_{\Delta}^{T}(s)\left(f(x(s))-f_{\Delta}\left(\bar{x}_{\Delta}(s)\right)\right)+\left|g(x(s))-g_{\Delta}\left(\bar{x}_{\Delta}(s)\right)\right|^{2}\right) d s \\
& \quad+\lambda \mathbb{E} \int_{0}^{t \wedge \theta}\left(\left|e_{\Delta}(s)+\left(h\left(x\left(s^{-}\right)\right)-h_{\Delta}\left(\bar{x}_{\Delta}\left(s^{-}\right)\right)\right)\right|^{2}-\left|e_{\Delta}(s)\right|^{2}\right) d s \\
& \leq \mathbb{E} \int_{0}^{t \wedge \theta}\left(2\left(x(s)-\bar{x}_{\Delta}(s)\right)^{T}\left(f(x(s))-f_{\Delta}\left(\bar{x}_{\Delta}(s)\right)\right)+\left|g(x(s))-g_{\Delta}\left(\bar{x}_{\Delta}(s)\right)\right|^{2}\right) d s+\bar{J}_{2} \\
& \quad+\mathbb{E} \int_{0}^{t \wedge \theta}\left(2 \lambda\left(x(s)-\bar{x}_{\Delta}(s)\right)^{T}\left(h\left(x\left(s^{-}\right)\right)-h_{\Delta}\left(\bar{x}_{\Delta}\left(s^{-}\right)\right)\right)+\lambda\left|h\left(x\left(s^{-}\right)\right)-h_{\Delta}\left(\bar{x}_{\Delta}\left(s^{-}\right)\right)\right|^{2}\right) d s+\bar{J}_{3} \\
& \leq \bar{L}_{1} \int_{0}^{t} \mathbb{E}\left|x(s \wedge \theta)-\bar{x}_{\Delta}(s \wedge \theta)\right|^{2} d s+\bar{J}_{2}+\bar{J}_{3},
\end{aligned}
$$

where

$$
\begin{array}{r}
\bar{J}_{2}=2 \mathbb{E} \int_{0}^{t \wedge \theta}\left|x_{\Delta}(s)-\bar{x}_{\Delta}(s) \| f(x(s))-f_{\Delta}\left(\bar{x}_{\Delta}(s)\right)\right| d s, \\
\bar{J}_{3}=2 \lambda \mathbb{E} \int_{0}^{t \wedge \theta}\left|x_{\Delta}(s)-\bar{x}_{\Delta}(s) \| h\left(x\left(s^{-}\right)\right)-h_{\Delta}\left(\bar{x}_{\Delta}\left(s^{-}\right)\right)\right| d s .
\end{array}
$$


By the condition $\mu^{-1}(\varphi(\Delta)) \geq n$ and the definition of the truncated functions $f_{\Delta}$ and $g_{\Delta}$, we have that

$$
f_{\Delta}\left(\bar{x}_{\Delta}(s)\right)=f\left(\bar{x}_{\Delta}(s)\right) \text { and } \quad g_{\Delta}\left(\bar{x}_{\Delta}(s)\right)=g\left(\bar{x}_{\Delta}(s)\right) \text {, for } 0 \leq s \leq t \wedge \theta \text {. }
$$

Hence, by Assumption 3.24 and the Hölder inequality as well as Lemma 3.19 and 3.20 , we get that

$$
\begin{aligned}
\bar{J}_{2} & \leq 2 \mathbb{E} \int_{0}^{t \wedge \theta}\left|x_{\Delta}(s)-\bar{x}_{\Delta}(s) \| f(x(s))-f\left(\bar{x}_{\Delta}(s)\right)\right| d s \\
& \leq 2 \bar{L}_{2} \mathbb{E} \int_{0}^{t \wedge \theta}\left|x_{\Delta}(s)-\bar{x}_{\Delta}(s)\left\|1+|x(s)|^{\bar{\gamma}}+\left|\bar{x}_{\Delta}(s)\right|^{\bar{\gamma}}\right\| x(s)-\bar{x}_{\Delta}(s)\right| d s \\
& \leq \bar{L}_{2} \int_{0}^{t \wedge \theta} \mathbb{E}\left|x(s)-\bar{x}_{\Delta}(s)\right|^{2} d s+C \int_{0}^{t \wedge \theta} \mathbb{E}\left(1+|x(s)|^{2 \bar{\gamma}}+\left|\bar{x}_{\Delta}(s)\right|^{2 \bar{\gamma}}\right)\left|x_{\Delta}(s)-\bar{x}_{\Delta}(s)\right|^{2} d s \\
& \leq \bar{L}_{2} \int_{0}^{t} \mathbb{E}\left|x(s \wedge \theta)-\bar{x}_{\Delta}(s \wedge \theta)\right|^{2} d s \\
& +C \int_{0}^{T}\left(1+\mathbb{E}|x(s)|^{2}+\mathbb{E}\left|\bar{x}_{\Delta}(s)\right|^{2}\right)^{\bar{\gamma}}\left(\mathbb{E}\left|x_{\Delta}(s)-\bar{x}_{\Delta}(s)\right|^{2 /(1-\bar{\gamma})}\right)^{1-\bar{\gamma}} d s \\
& \leq \bar{L}_{2} \int_{0}^{t} \mathbb{E}\left|x(s \wedge \theta)-\bar{x}_{\Delta}(s \wedge \theta)\right|^{2} d s+C(\varphi(\Delta))^{2} \Delta
\end{aligned}
$$

where condition $0 \leq \bar{\gamma}<1$ has been used. Similarly, we have

$$
\bar{J}_{3} \leq \lambda \bar{L}_{2} \int_{0}^{t} \mathbb{E}\left|x(s \wedge \theta)-\bar{x}_{\Delta}(s \wedge \theta)\right|^{2} d s+C(\varphi(\Delta))^{2} \Delta .
$$

Inserting 3.90, 3.91, into 3.89) and combining Lemma 3.19, we have

$$
\mathbb{E}\left|e_{\Delta}(t \wedge \theta)\right|^{2} \leq C \int_{0}^{t} \mathbb{E}\left|e_{\Delta}(s \wedge \theta)\right|^{2} d s+C(\varphi(\Delta))^{2} \Delta
$$

The Gronwall inequality complete the proof.

Theorem 3.26. Let Assumption $3.15,3.163 .23$ and 3.24 hold. Let $r \in(0,2)$. If

$$
\varphi(\Delta) \geq \mu\left(\bar{L}_{3}^{-(1+\bar{\gamma})}\left((\varphi(\Delta))^{r} \Delta^{r / 2}\right)^{-1 /(2-r)}\right)
$$

holds for all sufficiently small $\Delta \in(0,1]$, then for every such small $\Delta$,

$$
\mathbb{E}\left|x(T)-x_{\Delta}(T)\right|^{r} \leq C(\varphi(\Delta))^{r} \Delta^{r / 2}
$$

and

$$
\mathbb{E}\left|x(T)-\bar{x}_{\Delta}(T)\right|^{r} \leq C(\varphi(\Delta))^{r} \Delta^{r / 2},
$$

for any $T>0$. 
Proof. Let $\tau_{n}, \rho_{\Delta, n}, \theta_{\Delta, n}$ and $e_{\Delta}(t)$ be the same as before. By [3.83)-(3.85), inequality

$$
\mathbb{E}\left|e_{\Delta}(T)\right|^{r} \leq \mathbb{E}\left|e_{\Delta}\left(T \wedge \theta_{\Delta, n}\right)\right|^{r}+\frac{C r \delta}{2}+\frac{C(2-r)}{2 n^{2} \delta^{r /(2-r)}}
$$

holds for any $\Delta \in(0,1], n>\left|x_{0}\right|$ and $\delta>0$. We can therefore choose $\delta=(\varphi(\Delta))^{r} \Delta^{r / 2}$ and $n=\bar{L}_{3}^{-(1+\bar{\gamma})}\left((\varphi(\Delta))^{r} \Delta^{r / 2}\right)^{-1 /(2-r)}$ to get

$$
\mathbb{E}\left|e_{\Delta}(T)\right|^{r} \leq \mathbb{E}\left|e_{\Delta}\left(T \wedge \theta_{\Delta, n}\right)\right|^{r}+C(\varphi(\Delta))^{r} \Delta^{r / 2} .
$$

By condition 3.92, we have

$$
\mu^{-1}(\varphi(\Delta)) \geq \bar{L}_{4}^{-1+\bar{\gamma}}\left((\varphi(\Delta))^{r} \Delta^{r / 2}\right)^{-1 /(2-r)}=n .
$$

Using Lemma 3.25, we have

$$
\mathbb{E}\left|e_{\Delta}(T)\right|^{r} \leq\left(\mathbb{E}\left|e_{\Delta}(T)\right|^{2}\right)^{r / 2} \leq C\left((\varphi(\Delta))^{2} \Delta\right)^{r / 2}=C(\varphi(\Delta))^{r} \Delta^{r / 2} .
$$

125 Combining this with Lemma (3.19) gives 3.94). Thus, the proof is complete.

Corollary 3.27. Let Assumption $3.15,3.16,3.23$ and 3.24 hold. Define

$$
\mu(n)=\bar{L}_{3} n^{1+\bar{\gamma}}, \quad n \geq 0 .
$$

Let $0<r \leq 2 /(2+\bar{\gamma})$ and

$$
\varphi(\Delta)=\Delta^{-\varepsilon}, \quad \varepsilon \in\left[\frac{r(1+\bar{\gamma})}{4+2 r \bar{\gamma}}, \frac{1}{4}\right] .
$$

Assume that 3.92] holds for all sufficiently small $\Delta \in(0,1]$. Then,

$$
\mathbb{E}\left|x(T)-x_{\Delta}(T)\right|^{r} \leq C \Delta^{r(1-\varepsilon) / 2}
$$

and

$$
\mathbb{E}\left|x(T)-\bar{x}_{\Delta}(T)\right|^{r} \leq C \Delta^{r(1-\varepsilon) / 2} .
$$

Proof. Applying Theorem 3.26 along with 3.95 and 3.96 gives the required assertion 3.97) and (3.98).

Remark 3.28. Substituting (3.95) and (3.96) into 3.92) gives

$$
\Delta^{-\varepsilon} \geq \Delta^{-r(1-2 \varepsilon)(1+\bar{\gamma}) /(4-2 r)}, \quad \text { namely } \quad \varepsilon \geq \frac{r(1+\bar{\gamma})}{4+2 r \bar{\gamma}} .
$$

But, condition (3.96) means

$$
\frac{r(1+\bar{\gamma})}{4+2 r \bar{\gamma}} \leq \frac{1}{4}, \quad \text { namely } \quad r \leq \frac{2}{2+\bar{\gamma}} \leq 1 .
$$

Hence, we have to force $r$ to be not greater than $2 /(2+\bar{\gamma})$ in the corollary 3.27 
Remark 3.29. Fixing $0 \leq \bar{\gamma}<1$, by 3.96) and 3.97), we can conclude that convergence order is increasing in $\varepsilon$. Hence, substituting

$$
\varepsilon=\frac{r(1+\bar{\gamma})}{4+2 r \bar{\gamma}}
$$

into $r / 2(1-2 \varepsilon)$ obtains the optimal $\mathcal{L}^{r}$-convergence order, that is

$$
R:=\frac{r(2-r)}{2(2+r \bar{\gamma})}, \quad \text { for } \quad 0<r \leq \frac{2}{2+\bar{\gamma}},
$$

which means that convergence order $R$ increases as $r$ increases. In other words, the higher moment has a better convergence order for SDEs with jumps when $0<r \leq 2 /(2+\bar{\gamma})$. If we take

$$
r=\frac{2}{2+\bar{\gamma}}
$$

then 3.99 becomes

$$
R=\frac{1}{4+2 \bar{\gamma}},
$$

this is the maximum of optimal $\mathcal{L}^{r}$-convergence order. In particular, if $\bar{\gamma}=0$, i.e. the drift and the jump coefficients grow linearly, then convergence order is equal to $1 / 4$ by choosing $r=1$.

\section{Asymptotic behaviours}

\subsection{Stability}

In this subsection, we show that the partially truncated EM method can preserve the mean square exponential stability of the underlying SDE 2.1. For the purpose of stability, we also assume that

$$
f(0)=g(0)=h(0)=0,
$$

which means

$$
\left|F_{1}(x)\right| \vee\left|G_{1}(x)\right| \vee|h(x)| \leq K_{1}|x|, \quad \forall x \in \mathbb{R}^{d} .
$$

We first impose the following assumption.

Assumption 4.1. Assume that there exist constants $\theta \geq 0$ and $\alpha_{1}, \alpha_{2} \geq 0$ satisfying $\alpha_{1} \geq \alpha_{2}+$ $\lambda K_{1}\left(2+K_{1}\right)$ such that

$$
2 x^{T} F_{1}(x)+(1+\theta)\left|G_{1}(x)\right|^{2} \leq-\alpha_{1}|x|^{2}, \quad \forall x \in \mathbb{R}^{d},
$$

and

$$
2 x^{T} F(x)+\left(1+\theta^{-1}\right)|G(x)|^{2} \leq \alpha_{2}|x|^{2}, \quad \forall x \in \mathbb{R}^{d} .
$$

If there is no super-linearly growing term $G(x)$, we set $\theta=0$ and $\theta^{-1}|G(x)|^{2}=0$. Similarly, when the linearly growing term $G_{1}(x)$ is absent, we set $\theta=\infty$ and $\theta\left|G_{1}(x)\right|^{2}=0$. Moreover, this assumption means

$$
2 x^{T} f(x)+|g(x)|^{2}+\lambda\left(x^{T} h(x)+|h(x)|^{2}\right) \leq-\left(\alpha_{1}-\alpha_{2}-\lambda K_{1}\left(2+K_{1}\right)\right)|x|^{2}, \quad x \in \mathbb{R}^{d} .
$$

It is therefore known that the SDE 2.1 is exponentially stable in the mean square sense. We 
Lemma 4.2. Let Assumption 3.1 $3.2,3.3$ and 4.1 hold. Then for any initial value $x_{0} \in \mathbb{R}^{d}$, the solution of the SDE 2.1] satisfies

$$
\mathbb{E}|x(t)|^{2} \leq\left|x_{0}\right|^{2} e^{-\left(\alpha_{1}-\alpha_{2}-\lambda K_{1}\left(2+K_{1}\right)\right) t}, \quad \forall t \geq 0 .
$$

The following theorem shows that the truncated EM method preserves the mean square exponential stability perfectly. We employ the technique due to Guo et al. [16] to prove our results.

Theorem 4.3. Let Assumption 3.1 3.2, 3.3 and 4.1 hold. Then for any $\epsilon \in\left(0, \alpha_{1}-\alpha_{2}-\lambda K_{1}(2+\right.$ $\left.K_{1}\right)$ ), there exists a $\hat{\Delta} \in(0,1]$ such that for all $\Delta \in(0, \hat{\Delta}]$ and any initial value $x_{0} \in \mathbb{R}^{d}$, the truncated EM solutions satisfy

$$
\mathbb{E}\left|X_{\Delta}\left(t_{k}\right)\right|^{2} \leq\left|x_{0}\right|^{2} e^{-\left(\alpha_{1}-\alpha_{2}-\lambda K_{1}\left(2+K_{1}\right)-\epsilon\right) t_{k}}, \quad \forall k \geq 0 .
$$

Proof. Fix $\Delta \in(0,1]$. In the same way as Theorem 4.3 in [16] was proved, we have

$$
2 x^{T} f_{\Delta}(x)+\left|g_{\Delta}(x)\right|^{2} \leq-\left(\alpha_{1}-\alpha_{2}\right)|x|^{2}, \quad \forall x \in \mathbb{R}^{d} .
$$

From (3.14), we have

$$
\begin{aligned}
\mathbb{E}\left|X_{\Delta}\left(t_{k+1}\right)\right|^{2}= & \mathbb{E}\left(\left|X_{\Delta}\left(t_{k}\right)\right|^{2}+\left|f_{\Delta}\left(X_{\Delta}\left(t_{k}\right)\right)\right|^{2} \Delta^{2}+\left|g_{\Delta}\left(X_{\Delta}\left(t_{k}\right)\right) \Delta B_{k}\right|^{2}\right. \\
& +2 X_{\Delta}\left(t_{k}\right)^{T} f_{\Delta}\left(X_{\Delta}\left(t_{k}\right)\right) \Delta+\left|h\left(X_{\Delta}\left(t_{k}^{-}\right)\right) \Delta N_{k}\right|^{2} \\
& \left.+2 \Delta f_{\Delta}^{T}\left(X_{\Delta}\left(t_{k}\right)\right) h\left(X_{\Delta}\left(t_{k}^{-}\right)\right) \Delta N_{k}+2 X_{\Delta}\left(t_{k}\right)^{T} h\left(X_{\Delta}\left(t_{k}^{-}\right)\right) \Delta N_{k}\right),
\end{aligned}
$$

for $0 \leq k \leq M-1$. The property of Brownian increments implies

$$
\mathbb{E}\left|g_{\Delta}\left(X_{\Delta}\left(t_{k}\right)\right) \Delta B_{k}\right|^{2}=\Delta \mathbb{E}\left|g_{\Delta}\left(X_{\Delta}\left(t_{k}\right)\right)\right|^{2} .
$$

But, the Poisson increments satisfy $\mathbb{E} \Delta N_{k}=\lambda \Delta$ and $\mathbb{E}\left(\Delta N_{k}\right)^{2}=\lambda \Delta(1+\lambda \Delta)$. Hence, using the independence of the increments and [4.2], we find that

$$
\begin{aligned}
2 \mathbb{E}\left|X_{\Delta}\left(t_{k}\right) h\left(X_{\Delta}\left(t_{k}^{-}\right)\right) \Delta N_{k}\right| & \leq 2 K_{1} \mathbb{E}\left|X_{\Delta}\left(t_{k}\right)\right|^{2} \mathbb{E}\left|\Delta N_{k}\right|=2 K_{1} \lambda \Delta \mathbb{E}\left|X_{\Delta}\left(t_{k}\right)\right|^{2}, \\
\mathbb{E}\left|h\left(X_{\Delta}\left(t_{k}^{-}\right)\right) \Delta N_{k}\right|^{2} & \leq K_{1}^{2} \mathbb{E}\left|X_{\Delta}\left(t_{k}\right)\right|^{2} \mathbb{E}\left|\Delta N_{k}\right|^{2} \\
& \leq K_{1}^{2} \lambda \Delta(1+\lambda \Delta) \mathbb{E}\left|X_{\Delta}\left(t_{k}\right)\right|^{2} \\
& =K_{1}^{2} \lambda \Delta \mathbb{E}\left|X_{\Delta}\left(t_{k}\right)\right|^{2}+K_{1}^{2} \lambda^{2} \Delta^{2} \mathbb{E}\left|X_{\Delta}\left(t_{k}\right)\right|^{2}
\end{aligned}
$$

and

$$
\begin{aligned}
2 \mathbb{E}\left|\Delta f_{\Delta}\left(X_{\Delta}\left(t_{k}\right)\right) h\left(X_{\Delta}\left(t_{k}^{-}\right)\right) \Delta N_{k}\right| & \leq 2 K_{1} \Delta \mathbb{E}\left(\left|X_{\Delta}\left(t_{k}\right) f_{\Delta}\left(X_{\Delta}\left(t_{k}\right)\right)\right|\right) \mathbb{E}\left|\Delta N_{k}\right| \\
& \leq K_{1} \lambda \Delta^{2}\left(\mathbb{E}\left|X_{\Delta}\left(t_{k}\right)\right|^{2}+\mathbb{E}\left|f_{\Delta}\left(X_{\Delta}\left(t_{k}\right)\right)\right|^{2}\right) .
\end{aligned}
$$

Substituting (4.7)-(4.9) into (4.6) gives

$$
\begin{aligned}
\mathbb{E}\left|X_{\Delta}\left(t_{k+1}\right)\right|^{2} \leq & \mathbb{E}\left(\left|X_{\Delta}\left(t_{k}\right)\right|^{2}+2 X_{\Delta}\left(t_{k}\right)^{T} f_{\Delta}\left(X_{\Delta}\left(t_{k}\right)\right) \Delta+\left|g_{\Delta}\left(X_{\Delta}\left(t_{k}\right)\right)\right|^{2} \Delta\right) \\
& +\lambda K_{1}\left(2+K_{1}\right) \Delta \mathbb{E}\left|X_{\Delta}\left(t_{k}\right)\right|^{2}+\left(1+K_{1} \lambda\right) \Delta^{2} \mathbb{E}\left|f_{\Delta}\left(X_{\Delta}\left(t_{k}\right)\right)\right|^{2} \\
& +\left(K_{1}^{2} \lambda^{2}+K_{1} \lambda\right) \Delta^{2} \mathbb{E}\left|X_{\Delta}\left(t_{k}\right)\right|^{2} .
\end{aligned}
$$


By 4.5, we have

$$
\begin{aligned}
\mathbb{E}\left|X_{\Delta}\left(t_{k+1}\right)\right|^{2} \leq & {\left[1-\left(\alpha_{1}-\alpha_{2}-\lambda K_{1}\left(2+K_{1}\right)\right) \Delta\right] \mathbb{E}\left|X_{\Delta}\left(t_{k}\right)\right|^{2} } \\
& +\left(1+K_{1} \lambda\right) \Delta^{2} \mathbb{E}\left|f_{\Delta}\left(X_{\Delta}\left(t_{k}\right)\right)\right|^{2}+\left(K_{1}^{2} \lambda^{2}+K_{1} \lambda\right) \Delta^{2} \mathbb{E}\left|X_{\Delta}\left(t_{k}\right)\right|^{2} .
\end{aligned}
$$

By 3.1 and 4.1, we have

$$
\left|F_{\Delta}(x)\right|^{2} \leq 4 L_{1}|x|^{2}, \text { if }|x| \leq 1,
$$

and

$$
\left|F_{\Delta}(x)\right|^{2} \leq(\varphi(\Delta))^{2} \leq(\varphi(\Delta))^{2}|x|^{2}, \text { if }|x|>1 .
$$

Hence, we have

$$
\begin{aligned}
\Delta\left|f_{\Delta}(x)\right|^{2} & \leq 2\left(K_{1}^{2}+4 L_{1}+(\varphi(\Delta))^{2}\right) \Delta|x|^{2} \\
& \leq 2\left(\left(K_{1}^{2}+4 L_{1}\right) \Delta+\Delta^{1 / 2 \wedge(\bar{p}-2) / \bar{p}}\right)|x|^{2},
\end{aligned}
$$

for all $x \in \mathbb{R}^{d}$, where (3.10) has been used. For any $\epsilon \in\left(0, \alpha_{1}-\alpha_{2}-\lambda K_{1}\left(2+K_{1}\right)\right)$, there is a $\hat{\Delta} \in(0,1]$ sufficiently small such that for all $\Delta \in(0, \hat{\Delta}],\left(\alpha_{1}-\alpha_{2}-\lambda K_{1}\left(2+K_{1}\right)\right) \Delta \leq 1$ and

$$
\left\{\begin{array}{l}
2\left(1+K_{1} \lambda\right)\left(\left(K_{1}^{2}+4 L_{1}\right) \Delta+\Delta^{1 / 2 \wedge(\bar{p}-2) / \bar{p}}\right) \leq 0.5 \epsilon, \\
\left(K_{1}^{2} \lambda^{2}+K_{1} \lambda\right) \Delta \leq 0.5 \epsilon .
\end{array}\right.
$$

For each such $\Delta$, we have

$$
\left(1+K_{1} \lambda\right) \Delta^{2} \mathbb{E}\left|f_{\Delta}\left(X_{\Delta}\left(t_{k}\right)\right)\right|^{2}+\left(K_{1}^{2} \lambda^{2}+K_{1} \lambda\right) \Delta^{2} \mathbb{E}\left|X_{\Delta}\left(t_{k}\right)\right|^{2} \leq \epsilon \Delta \mathbb{E}\left|X_{\Delta}\left(t_{k}\right)\right|^{2} .
$$

Inserting this into 4.11 , we yield

$$
\begin{aligned}
\mathbb{E}\left|X_{\Delta}\left(t_{k+1}\right)\right|^{2} & \leq\left[1-\left(\alpha_{1}-\alpha_{2}-\lambda K_{1}\left(2+K_{1}\right)-\epsilon\right) \Delta\right] \mathbb{E}\left|X_{\Delta}\left(t_{k}\right)\right|^{2} \\
& \leq \cdots \\
& \leq\left|x_{0}\right|^{2}\left[1-\left(\alpha_{1}-\alpha_{2}-\lambda K_{1}\left(2+K_{1}\right)-\epsilon\right) \Delta\right]^{k+1} .
\end{aligned}
$$

By the elementary inequality

$$
1-\left(\alpha_{1}-\alpha_{2}-\lambda K_{1}\left(2+K_{1}\right)-\epsilon\right) \Delta \leq e^{-\left[\alpha_{1}-\alpha_{2}-\lambda K_{1}\left(2+K_{1}\right)-\epsilon\right] \Delta},
$$

we have

$$
\mathbb{E}\left|X_{\Delta}\left(t_{k+1}\right)\right|^{2} \leq\left|x_{0}\right|^{2} e^{-\left[\alpha_{1}-\alpha_{2}-\lambda K_{1}\left(2+K_{1}\right)-\epsilon\right] t_{k+1}} .
$$

Thus, the proof is complete.

\subsection{Asymptotic boundedness}

In this subsection, we show that the truncated EM method maintains the asymptotic boundedness of the underlying of SDE (2.1). The additional assumption is the following one. 
Assumption 4.4. Assume that there exist constants $\theta \geq 0$ and $\bar{\alpha}_{1}, \bar{\alpha}_{2}, \bar{\beta}_{1}, \bar{\beta}_{2}>0$ satisfying $\bar{\beta}_{1}>$ $\bar{\beta}_{2}+\max \left(\lambda\left(4 K_{1}^{2}+1\right), 2 \lambda K_{1}\left(2+K_{1}\right)\right)$ such that

$$
2 x^{T} F_{1}(x)+(1+\theta)\left|G_{1}(x)\right|^{2} \leq \bar{\alpha}_{1}-\bar{\beta}_{1}|x|^{2}, \quad \forall x \in \mathbb{R}^{d},
$$

and

$$
2 x^{T} F(x)+\left(1+\theta^{-1}\right)|G(x)|^{2} \leq \bar{\alpha}_{2}+\bar{\beta}_{2}|x|^{2}, \quad \forall x \in \mathbb{R}^{d} .
$$

When there is no super-linearly growing term $G(x)$, we set $\theta=0$ and $\theta^{-1}|G(x)|^{2}=0$. Similarly, if the linearly growing term $G_{1}(x)$ is absent, we set $\theta=\infty$ and $\theta\left|G_{1}(x)\right|^{2}=0$. Moreover, (3.3) implies

$$
\lambda\left(2 x^{T} h(x)+|h(x)|^{2}\right) \leq \lambda\left(|x|^{2}+2|h(x)|^{2}\right) \leq 4 \lambda K_{1}^{2}+\lambda\left(4 K_{1}+1\right)|x|^{2}, \quad \forall x \in \mathbb{R}^{d} .
$$

Hence, by Assumption 4.4, we have

$$
2 x^{T} f(x)+|g(x)|^{2}+\lambda\left(2 x^{T} h(x)+|h(x)|^{2}\right) \leq \hat{\alpha}-\hat{\beta}|x|^{2}, \quad \forall x \in \mathbb{R}^{d},
$$

where $\hat{\alpha}=\bar{\alpha}_{1}+\bar{\alpha}_{2}+4 \lambda K_{1}^{2}$ and $\hat{\beta}=\bar{\beta}_{1}-\bar{\beta}_{2}-\lambda\left(4 K_{1}^{2}+1\right)$.

Theorem 4.5. Let Assumption 3.1 3.23 .3 and 4.4 hold. Then for any initial value $x_{0} \in \mathbb{R}^{d}$, the solution of the SDE 2.1] satisfies

$$
\limsup _{t \rightarrow \infty} \mathbb{E}|x(t)|^{2} \leq \frac{\bar{\alpha}_{1}+\bar{\alpha}_{2}+4 \lambda K_{1}^{2}}{\bar{\beta}_{1}-\bar{\beta}_{2}-\lambda\left(4 K_{1}^{2}+1\right)} .
$$

Proof. Let $\tau_{n}, \hat{\alpha}$, and $\hat{\beta}$ be the same as before. Set $\sigma_{n}=t \wedge \tau_{n}$. For any $t \geq 0$, the Itô formula gives that

$$
\begin{aligned}
\mathbb{E}\left[e^{\hat{\beta} \sigma_{n}}\left|x\left(\sigma_{n}\right)\right|^{2}\right] & =\left|x_{0}\right|^{2}+\mathbb{E} \int_{0}^{\sigma_{n}} e^{\hat{\beta} \sigma_{n}}\left(2 x^{T}(s) f(x(s))+|g(x(s))|^{2}\right. \\
& \left.+2 x^{T}(s) h(x(s))+|h(x(s))|^{2}+\hat{\beta}|x(s)|^{2}\right) d s .
\end{aligned}
$$

By 4.15, we have

$$
\mathbb{E}\left[e^{\hat{\beta} \sigma_{n}}\left|x\left(\sigma_{n}\right)\right|^{2}\right] \leq\left|x_{0}\right|^{2}+\hat{\alpha} \int_{0}^{t} e^{\hat{\beta} s} d s=\left|x_{0}\right|^{2}+\frac{\hat{\alpha}}{\hat{\beta}}\left(e^{\hat{\beta} t}-1\right) .
$$

Letting $n \rightarrow \infty$, we have

$$
\mathbb{E}\left[e^{\hat{\alpha} t}|x(t)|^{2}\right] \leq\left|x_{0}\right|^{2}+\frac{\hat{\alpha}}{\hat{\beta}}\left(e^{\hat{\beta} t}-1\right)
$$

which implies

$$
\mathbb{E}|x(t)|^{2} \leq \frac{\left|x_{0}\right|^{2}}{e^{\hat{\beta} t}}+\frac{\hat{\alpha}}{\hat{\beta}}
$$

Thus, the proof is complete. 
Lemma 4.6. For $0<A<1$ and $B \geq 0$. If

$$
D_{k} \leq A D_{k-1}+B, \quad k=1,2, \cdots .
$$

Then

$$
\limsup _{k \rightarrow \infty} D_{k} \leq \frac{B}{1-A} .
$$

145 Proof. The proof is given in the Appendix.

Theorem 4.7. Let Assumption 3.1, 3.2, 3.3 and 4.4 hold. Then for any $\epsilon \in\left(0, \bar{\beta}_{1}-\bar{\beta}_{2}-\right.$ $\left.\max \left(\lambda\left(4 K_{1}^{2}+1\right), 2 \lambda K_{1}\left(2+K_{1}\right)\right)\right)$, there is $a \Delta \in(0,1]$ such that for every $\Delta \in(0, \hat{\Delta})$ and any initial value $x_{0} \in \mathbb{R}^{d}$, the truncated EM solutions satisfy

$$
\limsup _{k \rightarrow \infty} \mathbb{E}\left|X_{\Delta}\left(t_{k}\right)\right|^{2} \leq \frac{\bar{\alpha}_{1}+\bar{\alpha}_{2}+2 \lambda K_{1}\left(2+K_{1}\right)+\epsilon}{\bar{\beta}_{1}-\bar{\beta}_{2}-2 \lambda K_{1}\left(2+K_{1}\right)-\epsilon} .
$$

Proof. Fix $\varepsilon \in\left(0, \bar{\beta}_{1}-\bar{\beta}_{2}\right)$. In the same way as Theorem 5.3 in [16] was proved, we have

$$
2 x^{T} f_{\Delta}(x)+\left|g_{\Delta}(x)\right|^{2} \leq \bar{\alpha}_{1}+\bar{\alpha}_{2}-\left(\bar{\beta}_{1}-\bar{\beta}_{2}-0.5 \epsilon\right)|x|^{2}, \quad \forall x \in \mathbb{R}^{d},
$$

as long as $\Delta \in\left(0, \hat{\Delta}_{1}\right]$, where $\hat{\Delta}_{1} \in(0,1]$ is sufficiently small and satisfies

$$
\frac{\bar{\alpha}_{2}}{\left(\mu^{-1}\left(\varphi\left(\hat{\Delta}_{1}\right)\right)\right)^{2}} \leq 0.5 \epsilon
$$

Using the independence of the Poisson increments and 3.3 as well as Lemma 3.7, we have

$$
\begin{aligned}
\mathbb{E}\left|h\left(X_{\Delta}\left(t_{k}^{-}\right)\right) \Delta N_{k}\right|^{2} & \leq 2 K_{1}^{2} \mathbb{E}\left(1+\left|X_{\Delta}\left(t_{k}\right)\right|^{2}\right) \mathbb{E}\left|\Delta N_{k}\right|^{2} \\
& \leq 2 K_{1}^{2} \lambda \Delta(1+\lambda \Delta) \mathbb{E}\left(1+\left|X_{\Delta}\left(t_{k}\right)\right|^{2}\right) \\
& \leq 2 K_{1}^{2} \lambda \Delta \mathbb{E}\left|X_{\Delta}\left(t_{k}\right)\right|^{2}+2 K_{1}^{2} \lambda \Delta+C \Delta^{2}, \\
2 \mathbb{E}\left|X_{\Delta}\left(t_{k}\right) h\left(X_{\Delta}\left(t_{k}^{-}\right)\right) \Delta N_{k}\right| & \leq 2 K_{1} \mathbb{E}\left(\left|X_{\Delta}\left(t_{k}\right)\right|\left(1+\left|X_{\Delta}\left(t_{k}\right)\right|\right)\right) \mathbb{E}\left|\Delta N_{k}\right| \\
& \leq 4 K_{1} \lambda \Delta \mathbb{E}\left(1+\left|X_{\Delta}\left(t_{k}\right)\right|^{2}\right) \\
& \leq 4 K_{1} \lambda \Delta \mathbb{E}\left|X_{\Delta}\left(t_{k}\right)\right|^{2}+4 K_{1} \lambda \Delta
\end{aligned}
$$

and

$$
\begin{aligned}
2 \mathbb{E}\left|\Delta f_{\Delta}\left(X_{\Delta}\left(t_{k}\right)\right) h\left(X_{\Delta}\left(t_{k}^{-}\right)\right) \Delta N_{k}\right| & \leq 2 K_{1} \Delta \mathbb{E}\left(\left(1+\left|X_{\Delta}\left(t_{k}\right)\right|\right)\left|f_{\Delta}\left(X_{\Delta}\left(t_{k}\right)\right)\right|\right) \mathbb{E}\left|\Delta N_{k}\right| \\
& \leq K_{1} \lambda \Delta^{2}\left(\mathbb{E}\left(1+\left|X_{\Delta}\left(t_{k}\right)\right|^{2}\right)+\mathbb{E}\left|f_{\Delta}\left(X_{\Delta}\left(t_{k}\right)\right)\right|^{2}\right) \\
& \leq K_{1} \lambda \Delta^{2} \mathbb{E}\left|f_{\Delta}\left(X_{\Delta}\left(t_{k}\right)\right)\right|^{2}+C \Delta^{2} .
\end{aligned}
$$

Fix $x_{0} \in \mathbb{R}^{d}$ arbitrarily. For any $\Delta \in\left(0, \hat{\Delta}_{1}\right)$, substituting (4.22)-4.24) into 4.6 gives

$$
\begin{aligned}
\mathbb{E}\left|X_{\Delta}\left(t_{k+1}\right)\right|^{2} \leq & \mathbb{E}\left(\left|X_{\Delta}\left(t_{k}\right)\right|^{2}+2 X_{\Delta}\left(t_{k}\right)^{T} f_{\Delta}\left(X_{\Delta}\left(t_{k}\right)\right) \Delta+\left|g_{\Delta}\left(X_{\Delta}\left(t_{k}\right)\right)\right|^{2} \Delta\right) \\
& +2 \lambda K_{1}\left(2+K_{1}\right) \Delta \mathbb{E}\left|X_{\Delta}\left(t_{k}\right)\right|^{2}+\left(1+K_{1} \lambda\right) \Delta^{2} \mathbb{E}\left|f_{\Delta}\left(X_{\Delta}\left(t_{k}\right)\right)\right|^{2} \\
& +2 \lambda K_{1}\left(2+K_{1}\right) \Delta+C \Delta^{2} \\
\leq & \left(1-\left(\bar{\beta}_{1}-\bar{\beta}_{2}-2 \lambda K_{1}\left(2+K_{1}\right)-0.5 \varepsilon\right) \Delta\right) \mathbb{E}\left|X_{\Delta}\left(t_{k}\right)\right|^{2} \\
& +\left(\bar{\alpha}_{1}+\bar{\alpha}_{2}+2 \lambda K_{1}\left(2+K_{1}\right)\right) \Delta+C \Delta^{2} \\
& +\left(1+K_{1} \lambda\right) \Delta^{2} \mathbb{E} \mid f_{\Delta}\left(\left.X_{\Delta}\left(t_{k}\right)\right|^{2}\right.
\end{aligned}
$$


where 4.20 has been used. By 3.3 and 3.12 , we have

$$
\left|f_{\Delta}(x)\right|^{2} \leq 2\left|F_{1}(x)\right|^{2}+2|F(x)|^{2} \leq 4 K_{1}^{2}\left(1+|x|^{2}\right)+2(\varphi(\Delta))^{2}, \quad \forall x \in \mathbb{R}^{d} .
$$

Hence, by 3.10$)$, we get

$$
\Delta\left|f_{\Delta}(x)\right|^{2} \leq 4 K_{1}^{2} \Delta\left(1+|x|^{2}\right)+2 \Delta^{1 / 2 \wedge(\bar{p}-2) / \bar{p}}, \quad \forall x \in \mathbb{R}^{d} .
$$

Consequently, there is a $\hat{\Delta} \in\left(0, \hat{\Delta}_{1}\right)$ sufficiently small such that for any $\Delta \in(0, \hat{\Delta}), \Delta\left(\hat{\beta}_{1}-\hat{\beta}_{2}-\epsilon\right)<$ 1 and

$$
C \Delta+\left(1+K_{1} \lambda\right) \Delta\left|f_{\Delta}\left(X_{\Delta}\left(t_{k}\right)\right)\right|^{2} \leq \varepsilon+0.5 \epsilon\left|X_{\Delta}\left(t_{k}\right)\right|^{2} .
$$

Thus, fix any $\Delta \in(0, \hat{\Delta})$. Inserting 4.26 into 4.25 yields

$$
\begin{aligned}
\mathbb{E}\left|X_{\Delta}\left(t_{k+1}\right)\right|^{2} \leq & \left(1-\left(\bar{\beta}_{1}-\bar{\beta}_{2}-2 \lambda K_{1}\left(2+K_{1}\right)-\epsilon\right) \Delta\right) \mathbb{E}\left|X_{\Delta}\left(t_{k}\right)\right|^{2} \\
& +\left(\bar{\alpha}_{1}+\bar{\alpha}_{2}+2 \lambda K_{1}\left(2+K_{1}\right)+\epsilon\right) \Delta .
\end{aligned}
$$

Applying Lemma 4.6 to 4.27) gives the required assertion 4.19.

\section{Examples}

Example 5.1. Consider the scalar power logistic model in a population system with jumps

$$
d x(t)=x(t)\left[\left(5-10 x^{2}(t)\right)\right] d t+x^{2}(t) d B(t)+x\left(t^{-}\right) d N(t),
$$

with the initial value $x(0)=1$, where $B(t)$ is a scalar Brownian motion and $N(t)$ is a scalar Poisson process with intensity $\lambda=0.25$. Letting $h(x)=x$, we decompose $f(x)=5 x-10 x^{3}$ and $g(x)=x^{2}$ into two parts denoted by $f(x)=F_{1}(x)+F(x)$ and $g(x)=G_{1}(x)+G(x)$ with

$$
F_{1}(x)=5 x, \quad F(x)=-10 x^{3}, \quad G_{1}(x)=0, \quad G(x)=x^{2},
$$

respectively. We now demonstrate the process of implementing the truncated EM and show the convergence rate of this method for this system.

Step 1. Verify the assumptions.

Obviously, (3.1) is satisfied. It is easy to see that

$$
|F(x)-F(y)| \vee|G(x)-G(y)| \leq 15\left(1+x^{2}+y^{2}\right)|x-y|, \quad \forall x, y \in \mathbb{R} .
$$

Thus, Assumption 3.1 is satisfied with $\gamma=2$. Similarly, we can deduce that Assumption 3.2 and 3.3 is also fulfilled for $\bar{r}=3$ and $\bar{p}=21$, respectively.

Step 2. Choose $\mu(\cdot)$ and $\varphi(\cdot)$.

By 5.2, we have

$$
\sup _{|x| \leq n}(|F(x)| \vee|G(x)|) \leq 10 n^{3}, \quad \forall n \geq 1,
$$

which means $\mu(n)=10 n^{3}$. Setting $r=2$, then condition 3.61), namely, $(1+\gamma) r \vee \bar{r}<p<\bar{p}$, becomes $6<p<21$. If we let $p=20$ and choose a parameter $\varepsilon \in(0,1 / 4]$, say $\varepsilon=1 / 6$, then 3.64 , namely, $p \geq \frac{(1+\gamma) r}{2 \varepsilon}$, holds. Hence, according to 3.10, we can choose

$$
\varphi(\Delta)=10 \Delta^{-1 / 6} \text {. }
$$




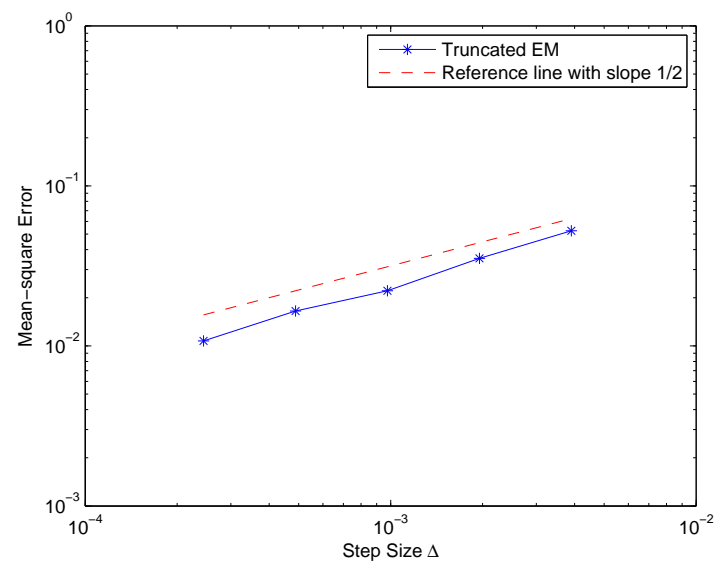

Fig. 1. The $\mathcal{L}^{1}$-convergence order of truncated EM scheme for SDE 5.1)

Step 3. Define $f_{\Delta}(x)$ and $g_{\Delta}(x)$.

From Step 2, we define the truncating factor $\mu^{-1}(\varphi(\Delta))=\Delta^{-1 / 18}$. The truncated functions $f_{\Delta}(x)$ and $g_{\Delta}(x)$ are defined by

$$
f_{\Delta}(x)=F_{1}(x)+F\left(\left(|x| \wedge \Delta^{-1 / 18}\right) \frac{x}{|x|}\right) \quad \text { and } \quad g_{\Delta}(x)=G_{1}(x)+G\left(\left(|x| \wedge \Delta^{-1 / 18}\right) \frac{x}{|x|}\right) .
$$

Step 4. Calculate $X_{k}$ in each iteration.

For the given step size $\Delta$, the time $T$ and $X_{0}=1$, the $X_{k+1}$ is calculated by

$$
X_{k+1}=X_{k}+f_{\Delta}\left(X_{k}\right) \Delta+g_{\Delta}\left(X_{k}\right) \Delta B_{k}+h\left(X_{k}\right) \Delta N_{k}, \quad 0 \leq k \leq T / \Delta-1 .
$$

For $p=20, \gamma=2, r=2$ and $\varepsilon=1 / 6$, we compute $\varepsilon(p-(1+\gamma) r) /(1+\gamma)=7 / 9,(p-\gamma r) / p=4 / 5$ and $r(1-2 \varepsilon) / 2=2 / 3$, respectively. By Theorem 3.11. we have

$$
\mathbb{E}\left|x(T)-x_{\Delta}(T)\right|^{2} \leq C \Delta^{2 / 3},
$$

which implies that the truncated EM method for SDE 5.1 has the order 2/3 of $\mathcal{L}^{2}$-convergence or the order $1 / 3$ of $\mathcal{L}^{1}$-convergence.

As the SDE (5.1) does not have any explicit solutions, the scheme 5.3 with step size $2^{-14}$ is treated as the true solution of SDE (5.1) in the numerical experiments. Fig. I] shows the $\mathcal{L}^{2}$-errors, which are defined by

$$
\left(\mathbb{E}\left|x(T)-x_{\Delta}(T)\right|^{2}\right)^{1 / 2} \approx\left(\frac{1}{1000} \sum_{i=1}^{1000}\left|[x(T)]^{i}-\left[x_{\Delta}(T)\right]^{i}\right|^{2}\right)^{1 / 2},
$$

150 with step sizes $2^{-11}, 2^{-10}, 2^{-9}, 2^{-8}$ and $2^{-7}$ at time $T=3$. For each step size, 1000 sample paths are simulated. The numerical simulation shows that the $\mathcal{L}^{1}$-convergence order of the partially truncated EM method for SDE (5.1) is approximately 1/2, which is close to the theoretical result obtained in this paper, see Fig. I for illustration. 
Example 5.2. Consider the following scalar SDE with jumps

$$
d x(t)=-\left(x(t)+x^{5}(t)\right) d t+x^{2}(t) d B(t)+x\left(t^{-}\right) d N(t)
$$

with the initial value $x(0)=0.5$, where $B(t)$ is a scalar Brownian motion and $N(t)$ is a scalar Poisson process with jump intensity $\lambda=0.5$. Obviously, we have

$$
F_{1}(x)=-x, \quad F(x)=-x^{5}, \quad G_{1}(x)=0, \quad G(x)=x^{2}, \quad h(x)=x,
$$

and

$$
\begin{aligned}
& \left|F_{1}(x)\right| \vee\left|G_{1}(x)\right| \vee|h(x)|=|x|, \quad \text { with } \quad K_{1}=1, \\
& |F(x)-F(y)| \vee|G(x)-G(y)| \leq L_{1}\left(1+x^{4}+y^{4}\right)|x-y|,
\end{aligned}
$$

where $L_{1}$ is a constant. This means that Assumption 3.1 is satisfied with $\gamma=4$. Setting $\theta=\infty$ gives

$$
2 x F_{1}(x)+(1+\theta)\left|G_{1}(x)\right|^{2}=-2 x^{2},
$$

and

$$
2 x F(x)+\left(1+\theta^{-1}\right)|G(x)|^{2}=-2 x^{6}+x^{4} \leq-2 x^{2}\left(x^{2}-\frac{1}{4}\right)^{2}+\frac{1}{8} x^{2} \leq \frac{1}{8} x^{2} .
$$

Hence, Assumption 4.1 is satisfied with $\alpha_{1}=2$ and $\alpha_{2}=1 / 8$. Moreover, for any $\bar{r}$, we have

$$
(x-y)(F(x)-F(y))+\frac{\bar{r}-1}{2}|G(x)-G(y)| \leq\left(1+\frac{(\bar{r}-1)^{2}}{4}\right)|x-y|^{2}, \quad \forall x \in \mathbb{R},
$$

which means that Assumption 3.2 is satisfied. Also, we can check that Assumption 3.3 holds for any $\bar{p}$ (see [16]). By Theorem 4.2, the SDE 5.4 is stable exponentially in the mean square sense for any initial value $x_{0} \in \mathbb{R}$ and the solution $x(t)$ of SDE 5.4 satisfies

$$
\mathbb{E}|x(t)|^{2} \leq\left|x_{0}\right|^{2} e^{-\left(\alpha_{1}-\alpha_{2}-\lambda K_{1}\left(2+K_{1}\right)\right) t}=\left|x_{0}\right|^{2} e^{-0.375 t}, \quad \forall t \geq 0 .
$$

From (5.5), we can choose $\mu(n)=n^{5}$ such that

$$
\sup _{|x| \leq n}(|F(x)| \vee|G(x)|)=\sup _{|x| \leq n}\left(\left|x^{5}\right| \vee\left|x^{2}\right|\right) \leq n^{5}, \quad \forall n \geq 1
$$

Letting $r=2, \bar{r}=3, \gamma=4, p=40$ and $\varphi(\Delta)=\Delta^{-1 / 8}$. Then we choose $\mu^{-1}(\varphi(\Delta))=\Delta^{-1 / 40}$. By Corollary 3.12 the numerical solutions converge strongly to the true solution in $\mathcal{L}^{2}$ with convergence order $[r(1-2 \varepsilon) / 2] \wedge[(p-\gamma r) / p]=3 / 4 \wedge 4 / 5=3 / 4$. Finally, by Theorem 4.3. for any $\epsilon \in(0,0.375)$, there exists a $\hat{\Delta} \in(0,1]$ such that for all $\Delta \in(0, \hat{\Delta}]$ and any initial value $x_{0} \in \mathbb{R}$, the solutions of the truncated EM method (3.14) satisfy

$$
\mathbb{E}\left|X_{\Delta}\left(t_{k}\right)\right|^{2} \leq\left|x_{0}\right|^{2} e^{-\left(\alpha_{1}-\alpha_{2}-\lambda K_{1}\left(2+K_{1}\right)-\epsilon\right) t_{k}}=\left|x_{0}\right|^{2} e^{-(0.375-\epsilon) t_{k}}, \quad \forall k \geq 0 .
$$

Figs. 2 and 3 demonstrate the mean square exponential stability of the truncated EM method.

Example 5.3. Consider the following scalar SDE with jumps

$$
d x(t)=\left(x(t)-x^{3}(t)\right) d t+x(t) d B(t)+x\left(t^{-}\right) d N(t)
$$




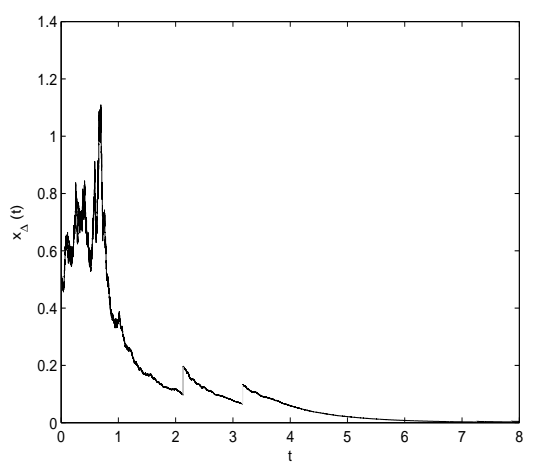

Fig. 2. A sample path of $x_{\Delta}(t)$ for (5.4) by truncated EM

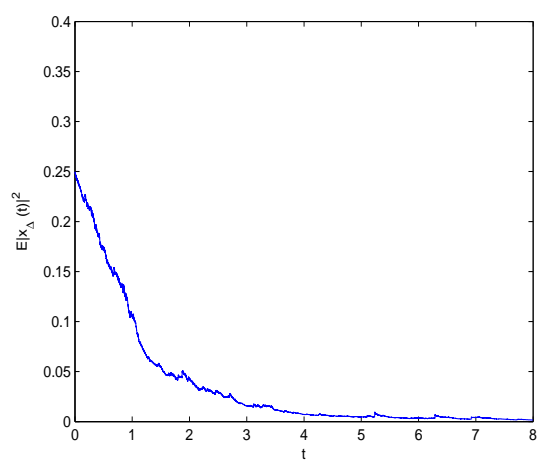

Fig. 3. Sample average of $x_{\Delta}^{2}(t)$ for $(5.4)$ by truncated EM with 1000 sample paths

with the initial value $x(0)=0.5$, where $B(t)$ is a scalar Brownian motion and $N(t)$ is a scalar Poisson process with jump intensity $\lambda=0.1$. We decompose the drift and diffusion coefficient in the form with

$$
F_{1}(x)=-2 x, \quad F(x)=3 x-x^{3}, \quad G_{1}(x)=x, \quad G(x)=0, \quad h(x)=x,
$$

which means

$$
\left|F_{1}(x)\right| \vee\left|G_{1}(x)\right| \vee|h(x)|=2|x|, \quad \text { with } \quad K_{1}=2
$$

Setting $\theta=0$ gives

$$
2 x F_{1}(x)+(1+\theta)\left|G_{1}(x)\right|^{2}=-3 x^{2},
$$

and

$$
2 x F(x)+\left(1+\theta^{-1}\right)|G(x)|^{2}=2 x\left(3 x-x^{3}\right)=-2\left(x^{2}-1.5\right)^{2}+4.5 \leq 4.5 .
$$

Hence, Assumption 4.4 is satisfied with

$$
\bar{\alpha}_{1}=0, \quad \bar{\beta}_{1}=3, \quad \bar{\alpha}_{2}=4.5, \quad \text { and } \quad \bar{\beta}_{2}=0 .
$$

It is easy to check that coefficients of the SDE 5.6 with their decompositions in 5.7 satisfy Assumption 3.1] 3.2 and 3.3 for any $\bar{p}>2$. Using Theorem 4.5 gives that for any initial value $x_{0} \in \mathbb{R}$, the solution $x(t)$ of SDE 5.6 satisfies

$$
\limsup _{t \rightarrow \infty} \mathbb{E}|x(t)|^{2} \leq \frac{\bar{\alpha}_{1}+\bar{\alpha}_{2}+4 \lambda K_{1}^{2}}{\bar{\beta}_{1}-\bar{\beta}_{2}-\lambda\left(4 K_{1}^{2}+1\right)} \approx 4.69 .
$$

Moreover, taking $r=2, \gamma=2, \bar{r}=3$ as well as $p=50$, we can choose $\mu(n)=4 n^{3}$ and $\varphi(\Delta)=4 \Delta^{-3 / 50}$ and to define the numerical solutions $X_{\Delta}\left(t_{k}\right)$ by the partially truncated EM method. By Theorem 3.11 this solutions of truncated EM converge to the true solution in $\mathcal{L}^{2}$ with convergence order $[r(1-2 \varepsilon) / 2] \wedge[(p-\gamma r) / p]=22 / 25 \wedge 23 / 25=0.88$. Finally, by Theorem 4.7 for any $\epsilon \in(0,1.3)$, there exists a $\hat{\Delta} \in(0,1]$ such that for all $\Delta \in(0, \hat{\Delta}]$ and any initial value $x_{0} \in \mathbb{R}$, the numerical solutions satisfy

$$
\limsup _{k \rightarrow \infty} \mathbb{E}\left|X_{\Delta}\left(t_{k}\right)\right|^{2} \leq \frac{\bar{\alpha}_{1}+\bar{\alpha}_{2}+2 \lambda K_{1}\left(2+K_{1}\right)+\epsilon}{\bar{\beta}_{1}-\bar{\beta}_{2}-2 \lambda K_{1}\left(2+K_{1}\right)-\epsilon}=\frac{6.1+\epsilon}{1.4-\epsilon} .
$$




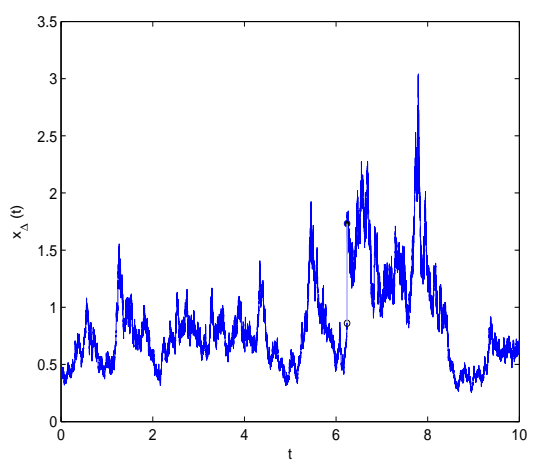

Fig. 4. A sample path of $x_{\Delta}(t)$ for 5.6 by truncated EM

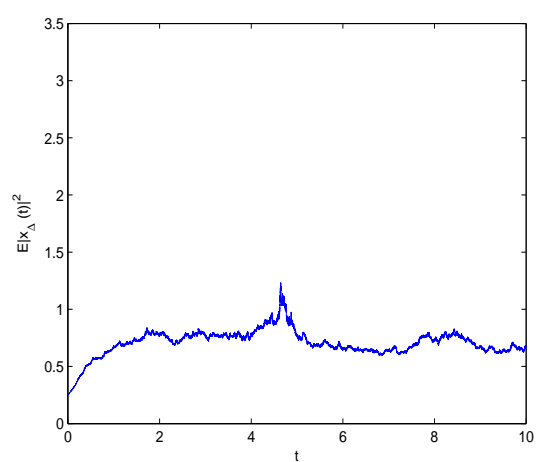

Fig. 5. Sample average of $x_{\Delta}^{2}(t)$ for 5.6 by truncated EM with 1000 sample paths

The asymptotic boundedness of the numerical method is shown in Figs. 4 and 5.

\section{Conclusions and future research}

In this paper, the truncated EM method is investigated for SDEs driven by both Brownian motions and Possion jumps. Both the finite time convergence and asymptotic behaviours of the method are studied. The $\mathcal{L}^{r}(r \geq 2)$ strong convergence is proved when the drift and diffusion coefficients satisfy super-linear growth condition and the coefficient for Possion jumps satisfies linear growth condition. When $0<r<2$, we are able to prove the $\mathcal{L}^{r}$-convergence of the methods to SDEs with all the three coefficients allowing to grow super-linearly.

In the future works, we will report on the SDEs driven by Lévy process and the $\mathcal{L}^{r}$-convergence for SDEs whose all the three coefficients can grow super-linearly.

\section{Appendix A. Proof of Lemma 3.9}

Proof. By the Itô formula and 3.8, we have

$$
\begin{aligned}
\mathbb{E}\left|x\left(t \wedge \tau_{n}\right)\right|^{2} \leq & \left|x_{0}\right|^{2}+\mathbb{E} \int_{0}^{t \wedge \tau_{n}} K_{3}\left(1+|x(s)|^{2}\right) d s \\
& +\lambda \mathbb{E} \int_{0}^{t \wedge \tau_{n}}\left(2 x(s)^{T} h(x(s))+|h(x(s))|^{2}\right) d s \\
\leq & \left|x_{0}\right|^{2}+\left(K_{3}+2 \lambda\left(2 K_{1}+K_{1}^{2}\right)\right) \int_{0}^{t} \mathbb{E}\left(1+\left|x\left(s \wedge \tau_{n}\right)\right|^{2}\right) d s,
\end{aligned}
$$

for any $0<t<T$. The Gronwall inequality shows

$$
\mathbb{E}\left|x\left(T \wedge \tau_{n}\right)\right|^{2} \leq C,
$$

which implies

Thus, the proof is complete.

$$
\mathbb{P}\left(\tau_{n} \leq T\right) \leq \frac{C}{n^{2}}
$$




\section{Appendix B. Proof of Lemma 3.10}

Proof. We write $\rho_{\Delta, n}=\rho$ for simplicity. For $0 \leq t \leq T$, the Itô formula gives

$$
\begin{aligned}
\mathbb{E}\left|x_{\Delta}(t \wedge \rho)\right|^{2}= & \left|x_{0}\right|^{2}+\mathbb{E} \int_{0}^{t \wedge \rho}\left(2 x_{\Delta}^{T}(s) f_{\Delta}\left(\bar{x}_{\Delta}(s)\right)+\left|g_{\Delta}\left(\bar{x}_{\Delta}(s)\right)\right|^{2}\right) d s \\
& +\lambda \mathbb{E} \int_{0}^{t \wedge \rho}\left(2 x_{\Delta}^{T}(s) h\left(\bar{x}_{\Delta}\left(s^{-}\right)\right)+\left|h\left(\bar{x}_{\Delta}\left(s^{-}\right)\right)\right|^{2}\right) d s \\
= & \left|x_{0}\right|^{2}+\mathbb{E} \int_{0}^{t \wedge \rho}\left(2 \bar{x}_{\Delta}^{T}(s) f_{\Delta}\left(\bar{x}_{\Delta}(s)\right)+\left|g_{\Delta}\left(\bar{x}_{\Delta}(s)\right)\right|^{2}\right) d s \\
& +\mathbb{E} \int_{0}^{t \wedge \rho} 2\left(x_{\Delta}(s)-\bar{x}_{\Delta}(s)\right)^{T} f_{\Delta}\left(\bar{x}_{\Delta}(s)\right) d s \\
& +\lambda \mathbb{E} \int_{0}^{t \wedge \rho}\left(2 x_{\Delta}^{T}(s) h\left(\bar{x}_{\Delta}\left(s^{-}\right)\right)+\left|h\left(\bar{x}_{\Delta}\left(s^{-}\right)\right)\right|^{2}\right) d s .
\end{aligned}
$$

By $(3.3)$, we obtain

$$
\begin{aligned}
& \mathbb{E} \int_{0}^{t \wedge \rho}\left(2 x_{\Delta}^{T}(s) h\left(\bar{x}_{\Delta}\left(s^{-}\right)\right)+\left|h\left(\bar{x}_{\Delta}\left(s^{-}\right)\right)\right|^{2}\right) d s \\
& \leq \mathbb{E} \int_{0}^{t \wedge \rho}\left(\left|x_{\Delta}(s)\right|^{2}+2\left|h\left(\bar{x}_{\Delta}\left(s^{-}\right)\right)\right|^{2}\right) d s \\
& \leq \mathbb{E} \int_{0}^{t \wedge \rho}\left(\left|x_{\Delta}(s)\right|^{2}+4 K_{1}^{2}\left(1+\left|\bar{x}_{\Delta}(s)\right|^{2}\right)\right) d s \\
& \leq 4 K_{1}^{2} T+\left(8 K_{1}^{2} T+1\right) \mathbb{E} \int_{0}^{t \wedge \rho}\left|x_{\Delta}(s)\right|^{2} d s+8 K_{1}^{2} T \mathbb{E} \int_{0}^{t \wedge \rho}\left|x_{\Delta}(s)-\bar{x}_{\Delta}(s)\right|^{2} d s .
\end{aligned}
$$

Substituting this into $(\mathrm{B.1})$ and applying 3.13 , we have

$$
\begin{aligned}
\mathbb{E}\left|x_{\Delta}(t \wedge \rho)\right|^{2} \leq & \left|x_{0}\right|^{2}+\int_{0}^{t \wedge \rho} 2 K_{4}\left(1+\left|\bar{x}_{\Delta}(s)\right|^{2}\right) d s+4 \lambda K_{1}^{2} T \\
& +\mathbb{E} \int_{0}^{t \wedge \rho} 2\left(x_{\Delta}(s)-\bar{x}_{\Delta}(s)\right)^{T} f_{\Delta}\left(\bar{x}_{\Delta}(s)\right) d s \\
& +\lambda\left(8 K_{1}^{2} T+1\right) \mathbb{E} \int_{0}^{t \wedge \rho}\left|x_{\Delta}(s)\right|^{2} d s+\lambda 8 K_{1}^{2} T \mathbb{E} \int_{0}^{t \wedge \rho}\left|x_{\Delta}(s)-\bar{x}_{\Delta}(s)\right|^{2} d s \\
\leq & \left(\left|x_{0}\right|^{2}+2 K_{4} T+4 \lambda L_{1}^{2} T\right)+\left(4 K_{4}+\lambda\left(8 K_{1}^{2} T+1\right)\right) \int_{0}^{t} \mathbb{E}\left|x_{\Delta}(s \wedge \rho)\right|^{2} d s \\
& +\left(4 K_{4}+8 \lambda K_{1}^{2} T\right) \int_{0}^{T} \mathbb{E}\left|x_{\Delta}(s)-\bar{x}_{\Delta}(s)\right|^{2} d s \\
& +2 \mathbb{E} \int_{0}^{t \wedge \rho}\left|x_{\Delta}(s)-\bar{x}_{\Delta}(s)\right|\left|f_{\Delta}\left(\bar{x}_{\Delta}(s)\right)\right| d s .
\end{aligned}
$$

By Lemma 3.6, we have

$$
\int_{0}^{T} \mathbb{E}\left|x_{\Delta}(s)-\bar{x}_{\Delta}(s)\right|^{2} d s \leq C .
$$


By 3.3 , we have

$$
\begin{aligned}
& \mathbb{E} \int_{0}^{t \wedge \rho}\left|x_{\Delta}(s)-\bar{x}_{\Delta}(s)\right|\left|f_{\Delta}\left(\bar{x}_{\Delta}(s)\right)\right| d s \\
& \leq K_{1} \mathbb{E} \int_{0}^{t \wedge \rho}\left|x_{\Delta}(s)-\bar{x}_{\Delta}(s)\right|\left(1+\left|\bar{x}_{\Delta}(s)\right|\right) d s+I_{5} \\
& \leq C\left(\mathbb{E} \int_{0}^{t \wedge \rho}\left|x_{\Delta}(s)-\bar{x}_{\Delta}(s)\right|^{2} d s+\int_{0}^{t} \mathbb{E}\left|x_{\Delta}(s \wedge \rho)\right|^{2} d s+1\right)+I_{5}
\end{aligned}
$$

where

$$
I_{5}=\mathbb{E} \int_{0}^{T}\left|x_{\Delta}(s)-\bar{x}_{\Delta}(s) \| F_{\Delta}\left(\bar{x}_{\Delta}(s)\right)\right| d s .
$$

Using Lemma 3.8, condition 3.10 and 3.12 gives

$$
\begin{aligned}
I_{5} & \leq \varphi(\Delta) \int_{0}^{T}\left(\mathbb{E}\left|x_{\Delta}(s)-\bar{x}_{\Delta}(s)\right|^{2}\right)^{1 / 2} d s \\
& \leq C(\varphi(\Delta))^{2} \Delta^{1 / 2}=C\left(\varphi(\Delta) \Delta^{1 / 4}\right)^{2} \leq C .
\end{aligned}
$$

Hence, we have

$$
\mathbb{E}\left|x_{\Delta}(t \wedge \rho)\right|^{2} \leq C\left(1+\int_{0}^{t} \mathbb{E}\left|x_{\Delta}(s \wedge \rho)\right|^{2} d s\right) .
$$

The Gronwall inequality gives

$$
\mathbb{E}\left|x_{\Delta}(T \wedge \rho)\right|^{2} \leq C,
$$

which implies 3.80. Thus, the proof is complete.

\section{Appendix C. Proof of Lemma 3.18}

Proof. Fix any $\Delta \in(0,1]$, we have

$$
\frac{1}{\mu^{-1}(\varphi(\Delta))} \leq \frac{1}{\mu^{-1}(\varphi(1))} .
$$

For $x \in \mathbb{R}^{d}$ with $|x| \leq \mu^{-1}(\varphi(\Delta))$, by the definition of the truncated function, we obtain the required assertion 3.71). For the case that $|x|>\mu^{-1}(\varphi(\Delta))$, Assumption 3.16 gives

$$
\begin{aligned}
& 2 x^{T} f_{\Delta}(x)+\left|g_{\Delta}(x)\right|^{2}+\lambda\left(2 x^{T} h_{\Delta}(x)+\left|h_{\Delta}(x)\right|^{2}\right) \\
= & 2\left(x-\pi_{\Delta}(x)\right)^{T} f_{\Delta}(x)+2 \lambda\left(x-\pi_{\Delta}(x)\right)^{T} h_{\Delta}(x) \\
& +2 \pi_{\Delta}(x)^{T} f_{\Delta}(x)+\left|g_{\Delta}(x)\right|^{2}+2 \lambda \pi_{\Delta}(x)^{T} h_{\Delta}(x)+\lambda\left|h_{\Delta}(x)\right|^{2} \\
\leq & \left(\frac{|x|}{\mu^{-1}(\varphi(\Delta))}-1\right)\left(2 \pi_{\Delta}(x)^{T} f\left(\pi_{\Delta}(x)\right)+2 \lambda \pi_{\Delta}(x)^{T} h\left(\pi_{\Delta}(x)\right)\right)+\bar{K}\left(1+\left|\pi_{\Delta}(x)\right|^{2}\right) \\
\leq & \left(\frac{|x|}{\mu^{-1}(\varphi(\Delta))}-1\right)\left(\bar{K}\left(1+\left|\pi_{\Delta}(x)\right|^{2}\right)\right)+\bar{K}\left(1+\left|\pi_{\Delta}(x)\right|^{2}\right)
\end{aligned}
$$




$$
\begin{aligned}
& =\frac{|x|}{\mu^{-1}(\varphi(\Delta))} \bar{K}\left(1+\left|\mu^{-1}(\varphi(\Delta))\right|^{2}\right) \\
& =\bar{K}|x|\left(\frac{1}{\mu^{-1}(\varphi(\Delta))}+\left|\mu^{-1}(\varphi(\Delta))\right|\right) \\
& \leq \bar{K}\left(\frac{1}{\mu^{-1}(\varphi(\Delta))} \vee 1\right)|x|(1+|x|) \\
& \leq 2 \bar{K}\left(\frac{1}{\mu^{-1}(\varphi(\Delta))} \vee 1\right)\left(1+|x|^{2}\right) .
\end{aligned}
$$

Thus, we complete the proof.

\section{Appendix D. Proof of Lemma 4.6}

Proof. 4.17) is equivalent to the following expression

$$
D_{k}+\frac{B}{A-1} \leq A\left(D_{k-1}+\frac{B}{A-1}\right), \quad \text { for } \quad k=0,1,2, \cdots .
$$

Hence, we have

$$
D_{k}+\frac{B}{A-1} \leq A^{k}\left(D_{0}+\frac{B}{A-1}\right)
$$

It follows

$$
D_{k} \leq A^{k}\left(D_{0}+\frac{B}{A-1}\right)+\frac{B}{1-A} .
$$

Recalling $0<A<1$ and taking $k \rightarrow \infty$, we obtain the required assertion (4.18).

\section{Acknowledgment}

This work was supported by the National Natural Science Foundation of China (Nos. 71571001,

61703003, 11701378, 11871343), Shanghai Education Development Foundation and Shanghai Municipal Education Commission (16CG50).

\section{References}

[1] E. Allen, Modeling with Itô Stochastic Differential Equations, Springer, Dordrecht, 2007.

[2] X. Mao, Stochastic Differential Equations and Applications, Horwood, 2nd edition, 2008.

180 [3] S. Deng, W. Fei, Y. Liang, X. Mao, Convergence of the split-step $\theta$-method for stochastic age-dependent population equations with Markovian switching and variable delay, Appl. Numer. Math. (2019) http://doi.org/10.1016/ j.apnum.2018.12.014.

[4] D. J. Higham, P. E. Kloeden, Numerical methods for nonlinear stochastic differential equations with jumps, Numer. Math. 101 (2005) 101-119.

[5] D. J. Higham, P. E. Kloeden, Convergence and stability of implicit methods for jump-diffusion systems, Int. J. Numer. Anal. Model. 3 (2006) 125-140.

[6] D. J. Higham, P. E. Kloeden, Strong convergence rates for backward Euler on a class of nonlinear jump-diffusion problems, J. Comput. Appl. Math. 205 (2007) 949-956.

[7] K. Dareiotis, C. Kumar, S. Sabanis, On tamed Euler approximations of SDEs driven by Lévy noise with applications to delay equations, SIAM J. Numer. Anal. 54 (2016) 1840-1872. 
[8] C. Kumar, S. Sabanis, On tamed Milstein schemes of SDEs driven by Lévy noise, Discrete Contin. Dyn. Syst. Ser. B 22 (2017) 421-463.

[9] M. Hutzenthaler, A. Jentzen, P. E. Kloeden, Strong convergence of an explicit numerical method for SDEs with nonglobally Lipschitz continuous coefficients, Ann. Appl. Probab. 22 (2012) 1611-1641.

195 [10] D. J. Higham, Stochastic ordinary differential equations in applied and computational mathematics, IMA J. Appl. Math. 76 (2011) 449-474.

[11] Z. Zhang, H. Ma, Order-preserving strong schemes for SDEs with locally Lipschitz coefficients, Appl. Numer. Math. 112 (2017) 1-16.

[12] S. Sabanis, A note on tamed Euler approximations, Electron. Commun. Probab. 18 (2013) 1-10.

200 [13] M. Hutzenthaler, A. Jentzen, Numerical approximations of stochastic differential equations with non-globally Lipschitz continuous coefficients, Mem. Amer. Math. Soc. 236 (2015) 1-95.

[14] X. Mao, The truncated Euler-Maruyama method for stochastic differential equations, J. Comput. Appl. Math. 290 (2015) 370-384.

[15] X. Mao, Convergence rates of the truncated Euler-Maruyama method for stochastic differential equations, J. Comput. Appl. Math. 296 (2016) 362-375.

[16] Q. Guo, W. Liu, X. Mao, R. Yue, The partially truncated Euler-Maruyama method and its stability and boundedness, Appl. Numer. Math. 115 (2017) 235-251.

[17] W. Zhang, M. H. Song, M. Z. Liu, Strong convergence of the partially truncated Euler-Maruyama method for a class of stochastic differential delay equations, J. Comput. Appl. Math. 335 (2018) 114-128.

210 [18] L. Tan, C. Yuan, Convergence rates of truncated EM scheme for NSDDEs, arXiv:1801.05952v1 (2018)

[19] C. Kumar, S. Sabanis, On explicit approximations for Lévy driven SDEs with super-linear diffusion coefficients, Electron. J. Probab. 22 (2017) 1-19.

[20] X. Yang, X. Wang, A transformed jump-adapted backward Euler method for jump-extended CIR and CEV models, Numer. Algorithms 74 (2017) 39-57.

215 [21] P. Przybyl owicz, Optimal global approximation of stochastic differential equations with additive Poisson noise, Numer. Algorithms 73 (2016) 323-348.

[22] W. Mao, S. You, X. Mao, On the asymptotic stability and numerical analysis of solutions to nonlinear stochastic differential equations with jumps, J. Comput. Appl. Math. 301 (2016) 1-15.

[23] X. Wang, S. Gan, Compensated stochastic theta methods for stochastic differential equations with jumps, Appl. Numer. Math. 60 (2010) 877-887.

[24] P. E. Kloeden, E. Platen, Numerical Solution of Stochastic Differential Equations, Springer-Verlag, Berlin, 1992.

[25] E. Platen, N. Bruti-Liberati, Numerical Solution of Stochastic Differential Equations with Jumps in Finance, Springer-Verlag, Berlin, 2010.

[26] L. Hu, X. Li, X. Mao, Convergence rate and stability of the truncated Euler-Maruyama method for stochastic differential equations, J. Comput. Appl. Math. 337 (2018) 274-289.

[27] J. Bao, B. Böttcher, X. Mao, C. Yuan, Convergence rate of numerical solutions to SFDEs with jumps, J. Comput. Appl. Math. 236 (2011) 119-131.

[28] Q. Guo, W. Liu, X. Mao, A note on the partially truncated Euler-Maruyama method, Appl. Numer. Math. 130 (2018) 157-170. 